FinAL VERsion - Cid@JHU - June/25/2000

Preprint typeset using $\mathrm{LAT}_{\mathrm{E} X}$ style emulateapj v. 20/04/00

\title{
QUASAR VARIABILITY IN THE FRAMEWORK OF POISSONIAN MODELS
}

\author{
R. Cid FERnANDEs ${ }^{1}$ \\ Department of Physics \& Astronomy, Johns Hopkins University, Baltimore, MD, 21218 \\ L. SODRÉ JR. \\ Instituto Astrônomico e Geofísico da USP, Av. Miguel Stefano 4200, 04301-904 São Paulo, SP, Brazil \\ AND \\ L. Vieira DA Silva JR. \\ Departamento de Física - CFM, Universidade Federal de Santa Catarina, C.P 476, 88040-900 Florianópolis, \\ SC, Brazil \\ Final version - Cid@JHU - June/25/2000
}

\begin{abstract}
Quasar optical monitoring campaigns are reaching a new standard of quality as a result of long term, accurate CCD observations. In this paper we review the basic Poissonian formulation of quasar variability, using it as a mathematical tool to extract relevant parameters such as the energy, rate and lifetimes of the flares through the analysis of observed light curves. It is shown that in this very general framework the well established anti-correlation between variability amplitude and wavelength can only be understood as an effect of an underlying spectral component which remains stable on long time-scales, and is redder than the variable component. The formalism is applied to the $\mathrm{B}$ and $\mathrm{R}$ light curves of 42 PG quasars collected by the Wise Observatory group (Giveon et al. 1999). Variability indices for these data are obtained with a Structure Function analysis. The mean number of living flares is constrained to be in the range between $\mathcal{N} \sim 5$ and 100, while their rates are found to be of order $\nu \sim 1-100$ $\mathrm{yr}^{-1}$. Monochromatic optical flare energies $E_{\lambda} \sim 10^{46-48} \mathrm{erg} \AA^{-1}$ and life-times $\tau$ of $\sim 0.5$ to $3 \mathrm{yr}$ are derived. Lower limits of typically $25 \%$ are established for the contribution of a non-variable component in the $\mathrm{R}$ band. The substantial diversity in these properties among quasars invalidates simple versions of the Poissonian model in which flare energies, lifetimes and the background contribution are treated as universal invariants. Light Curve simulations confirm the applicability of the method. Few significant correlations between variability indices and multi-wavelength properties of the quasars exist, confirming the results of Giveon et al. The good correlation between the $\mathrm{EW}(\mathrm{H} \beta)$ and the long term variability amplitude is interpreted in a scenario where only the variable component participates in the ionization of the line emitting gas. This idea is consistent with the observed trends of the variability amplitude with $\lambda, \mathrm{EW}(\mathrm{HeII})$ and the X-ray to optical spectral index. The parameter estimates derived under the framework of Poissonian models are applicable to several scenarios for the nature of quasar variability, and can help guiding, testing and discriminating between detailed physical models.
\end{abstract}

Subject headings: quasars: general - galaxies:active - galaxies:Seyfert - galaxies: nuclei - methods: statistical - methods: analytical

\section{INTRODUCTION}

Variability is one of the defining properties of Active Galactic Nuclei (AGN) and a potentially powerful discriminant among different scenarios for the physics of the central engine. Optical-UV variability studies in the past decade have put a lot of effort into investigating the effects of continuum variations upon the emission lines, leading to substantial progress in the diagnostics of the properties of the Broad Line Region (Netzer \& Peterson 1997). Yet, no comparable progress has been achieved in understanding the origin of the continuum fluctuations.

In this paper we discuss quasar variability in the context of Poissonian models. By Poissonian we mean any physical system in which the variations are due to the stochastic superposition of independent flares, occurring at a given mean rate but randomly distributed in time, a process also called "christmas-tree", "shot-noise", "discrete events" or "subunits" model. This approach has two key advantages:
(1) It provides a simple mathematical framework which can be applied to observed light curves to constrain the most relevant flare properties, such as energy, time-scale and rate (e.g., Cid Fernandes, Aretxaga \& Terlevich 1996). (2) It encompasses a large class of physical models for AGN. For instance, scenarios as diverse as accretion-disk instabilities of several kinds (Haardt, Maraschi \& Ghisellini 1994; Kawaguchi et al. 1998), disruption of stars in the gravitational field of super-massive black-hole (Peterson \& Ferland 1986; Ayal, Livio \& Piran 2000), stellar collisions (Keenan 1978; Courvoisier, Paltani \& Walter 1996), supernovae (Aretxaga \& Terlevich 1994; Aretxaga, Cid Fernandes \& Terlevich 1997), and even extrinsic variability models such as micro-lensing (Hawkings 2000) all share a common Poissonian nature. It is the combination of these two points that makes the Poissonian interpretation of AGN variability attractive, as its application to observed data may provide feasibility tests on different theories.

\footnotetext{
${ }^{1}$ Gemini Fellow. On leave of absence from Depto. de Física - CFM, UFSC, Florianópolis, SC, Brazil
} 
The early realization that most luminous sources tend to vary less (Uomoto, Wills \& Wils 1976; Pica \& Smith 1983) qualitatively favored a Poissonian interpretation. However, these and subsequent quasar monitoring studies found that the slope $(\beta)$ of the fractional variability $(\delta \equiv \sigma(L) / \bar{L})$ versus mean luminosity relation, $\delta \propto \bar{L}^{\beta}$, is shallower than the $\beta=-1 / 2$ value predicted in a simple Poissonian model (Cristiani, Vio \& Andreani 1990; Trevese et al. 1994; Hook et al. 1994, Cristiani et al. 1996, Paltani \& Courvoisier 1997), which lead some of these studies to rule out such models. Some studies, however, find slopes consistent with $\beta=-1 / 2$ (Cid Fernandes et al. 1996; Garcia et al. 1999), while others even question the very existence of a correlation (Bonoli et al. 1979; Netzer \& Sheffer 1983; Giallongo, Trevese \& Vagnetti 1991; Lloyd 1984; Cimatti, Zamorani \& Marano 1993; Netzer et al. 1996).

The slope of the variability-luminosity relation has thus been a controversial issue, with conflicting results reported in the literature. Selection effects and different coverages of the luminosity-redshift plane are likely causes for these discrepancies, as discussed in Hook et al. (1994). Garcia etal. (1999) argued that, although the observed variability-luminosity relation in their sample is shallower than $\beta=-1 / 2$, good agreement between data and model is achieved after taking into account the increase in variability with frequency. Moreover, Cid Fernandes et al. (1996) argued that the photometric (at least for photographic data) and sampling uncertainties, along with wavelength/redshift effects, propagate to a poorly defined variability-luminosity relation. Furthermore, they showed that a slope of $-1 / 2$ is only expected in the simplest of Poissonian models, in which the flare energy, time-scale and background contribution are held fixed as universal constants for all objects. Discarding Poissonian models on the basis of $\beta \neq-1 / 2$ is thus both risky and an oversimplified interpretation of Poissonian processes.

Quasar variability studies are reaching a new level of quality due to the efforts of several groups which engaged into long term, differential CCD photometric and spectroscopic monitoring programs (Borgeest \& Schramm 1994; Netzer et al. 1996; Sirola et al. 1998; Giveon et al. 1999hereinafter G99; Garcia etal. 1999; Kaspi et al. 2000). These studies represent an enormous improvement over pre-existing variability databases, both in sampling and photometric quality. While most previous studies (by necessity) based their analysis on properties of the ensemble of objects, these new data allow the study of quasar variability properties on an object-by-object basis. With the continuation and steady improvement of these programs, a clearer picture of the phenomenology of quasar variability will eventually emerge.

In this paper we review the formal relations between observable variability properties and the parameters in a general Poissonian model (§2). This approach is here seen as a valid step towards a physical understanding of the nature of AGN variability, which is particularly relevant given the current lack of an accepted paradigm for this ubiquitous phenomenon. Emphasis is put on the use of multi-wavelength data to constrain the basic model parameters. In $\S 3$ we apply the formalism to the photometric ( $\mathrm{B}$ and $\mathrm{R}$ ) quasar monitoring data collected over the past decade in the Wise Observatory (G99), by means of basic light curve statistics and an analysis of the individual Structure Functions. Simulations are used to verify the consistency of the results. A discussion on the use of higher moments of the data (skewness and kurtosis) is also presented (Appendix A). In Section 4 we discuss our results and present a correlation analysis of the variability with other properties, as well as possible interpretations in the context of AGN models. Finally, in Section 5 we summarize our main results and outline prospects of future work.

\section{FORMALISM}

Poissonian models for AGN variability have been studied both qualitatively and quantitatively, and for wavelengths across the electromagnetic spectrum, from radio (e.g., Dent 1972), to optical-UV (e.g., Cid Fernandes et al. 1996; Garcia et al. 1999) and X-rays (e.g., Lehto 1989; Almaini et al. 2000). In this section we follow the detailed formulation of this problem developed by Cid Fernandes (1995). The resulting formulae rest upon basic probability and time-series theory (e.g., Papoulis 1965).

In a Poissonian scenario the instantaneous monochromatic luminosity $L_{\lambda}(t)$ is due to the superposition of a variable component, $V_{\lambda}(t)$, and an underlying background component $C_{\lambda}$ :

$$
L_{\lambda}(t)=V_{\lambda}(t)+C_{\lambda}
$$

where $V_{\lambda}(t)$ is made by the superpositions of flares $l_{\lambda}(t)$ with random "birth-dates" $t_{i}$ :

$$
V_{\lambda}(t)=\sum_{i} l_{\lambda}\left(t-t_{i}\right)
$$

The first two moments (mean luminosity and relative variability) of the variable component are

$$
\begin{gathered}
\overline{V_{\lambda}}=\nu_{\lambda} E_{\lambda} \\
\frac{\sigma\left(V_{\lambda}\right)}{\overline{V_{\lambda}}}=\frac{1}{\left(\nu_{\lambda} \tau_{\lambda}\right)^{1 / 2}}
\end{gathered}
$$

In these expressions $\nu_{\lambda}$ is the mean rate of flares, $E_{\lambda}$ is the monochromatic energy of individual flares, and $\tau_{\lambda}$ is the flare "life-time", defined by

$$
\tau_{\lambda} \equiv \frac{E_{\lambda}^{2}}{\int l_{\lambda}^{2}(t) d t}
$$

It can be shown that these relations also hold when one allows for the (likely) possibility that the flares within a given object are not all identical, i.e., when $l_{\lambda}(t)=l_{\lambda}(t, \mathbf{x})$, where $\mathbf{x}$ denotes a general set of parameters (size, density, cooling time, ...). The only modifications in this more general case is that $E_{\lambda}$ above means the average $E_{\lambda}(\mathbf{x})$ over the probability distribution of $\mathbf{x}$, and similarly for $\tau_{\lambda}{ }^{2}$

${ }^{2}$ To be precise, if $p(\mathbf{x})$ is the probability density of $\mathbf{x}$, one finds

$$
\tau_{\lambda} \equiv{\overline{E_{\lambda}}}^{2} / \iint l_{\lambda}^{2}(t, \mathbf{x}) p(\mathbf{x}) d \mathbf{x} d t
$$


The total mean luminosity and relative variability are both affected by the underlying constant component, turning eqs. (3) and (4) into

$$
\begin{gathered}
\overline{L_{\lambda}}=\nu_{\lambda} E_{\lambda}+C_{\lambda} \\
\delta_{\lambda} \equiv \frac{\sigma\left(L_{\lambda}\right)}{\overline{L_{\lambda}}}=v_{\lambda} \frac{1}{\left(\nu_{\lambda} \tau_{\lambda}\right)^{1 / 2}}
\end{gathered}
$$

where $v_{\lambda} \equiv \overline{V_{\lambda}} / \overline{L_{\lambda}}$ is the fraction of the mean luminosity which is actually due to the variable component. Allowance for the possible contribution of a non-variable component is essential, though it has often been forgotten when discussing Poissonian models. Physically, $C_{\lambda}$ can be associated with several sources. These can be either around the nucleus and extrinsic to the variability generation process, as the host galaxy stellar contribution, or, more interestingly, a part of the variable continuum source which remains stable over long time-scales, such as the non-flaring part of an accretion disk.

The Poissonian model thus involves four basic parameters: the rate $\left(\nu_{\lambda}\right)$, energy $\left(E_{\lambda}\right)$ and life-time $\left(\tau_{\lambda}\right)$ of the flares, plus $C_{\lambda}$. The shape of the flares may be regarded as a further degree of freedom, but it has little effect upon the analysis presented here. Equations (6) and (7) relate these parameters to just two observables. Even estimating $\tau_{\lambda}$ through a Structure Function (SF) analysis, one has a non-closed system, with three observables and four variables. Higher moments of the light curve could in principle be used as further constraints (see Appendix A), but these are so badly affected by sampling uncertainties that presently they do not provide useful constraints. Another piece of information that can help constraining the model parameters is that the constant component has to be at most as strong as the observed minimum in the light curve:

$$
0 \leq C_{\lambda} \leq L_{\lambda, \min }
$$

It is reasonable to presume that the same flares are seen across a narrow spectral band, such as the optical-UV. Empirical support for this hypothesis comes from the high similarity between the continuum light curves in different bands (e.g., Cutri et al. 1985; Krolik et al. 1991; G99). Indeed, the whole method of reverberation mapping relies on an equivalent hypothesis, namely, that the fluctuations in the ionizing continuum can be mapped by those of the optical-UV continuum (Peterson 1993). We therefore may write $\nu_{\lambda}=\nu$ and $\tau_{\lambda}=\tau$. The developments below could also be made allowing for $\lambda$ dependent time-scales, but we shall adopt the simpler constant $\tau_{\lambda}$ scenario.

A first consequence of this assumption is that one can isolate the spectral shape of flare energy directly from the spectral behavior of the standard deviation $\sigma_{\lambda}$ :

$$
E_{\lambda}=\left(\frac{\tau}{\nu}\right)^{1 / 2} \sigma_{\lambda} \propto \sigma_{\lambda}
$$

It is well established that the amplitude of the variations increase towards shorter wavelengths (Cutri et al. 1985; Edelson, Krolik \& Pike 1990; Kinney et al. 1991; Cristiani et al. 1997; Di Clemente et al. 1996), with most sources becoming bluer as they brighten, which immediately tells us that the variable component is blue. This fact also has the very interesting consequence (see eq. [7]) that $v_{\lambda}$ must vary with wavelength, which can only be understood invoking an underlying component.

Another way of seeing this is to rewrite (7) as $\delta_{\lambda}=$ $v_{\lambda} \mathcal{N}^{-1 / 2}$, where $\mathcal{N}=\nu \tau$ is the mean number of living flares at any time. Since we are assuming that the same flares are seen in different wavebands, the fact that $\delta_{\lambda}$ decreases towards the red can only be made consistent with a Poissonian scenario if the relative contribution of the background,

$$
c_{\lambda} \equiv \frac{C_{\lambda}}{\overline{L_{\lambda}}}=1-v_{\lambda},
$$

increases with $\lambda$. Of course, the decomposition of opticalUV spectra of AGN into a variable plus a constant component has been proposed before, both on observational and on theoretical grounds. Here we proved that the spectral behavior of $\delta_{\lambda}$ implies the existence of a constant source if one is to keep within the framework of Poissonian models.

One can construct families of possible spectra for the constant component by writing

$$
C_{\lambda}=\overline{L_{\lambda}}-\mathcal{N}^{1 / 2} \sigma_{\lambda}
$$

The simultaneous analysis of the light curve statistics in different wavebands thus has interesting consequences, but we still do not have a closed system. Except for the life-time $\tau$, which can be estimated through a structure function analysis, there is no way to determine absolute values for $\nu, E_{\lambda}$ or $C_{\lambda}$. Nonetheless, the wavelength information, coupled with the condition that $C_{\lambda}$ must satisfy (8), can yield improved constraints upon $\mathcal{N}$. One may combine the above relations to obtain

$$
\left(\frac{1-\mu_{\lambda}}{\delta_{\lambda}}\right)^{2} \leq \mathcal{N} \leq\left(\frac{1}{\delta_{\lambda}}\right)^{2}
$$

where $\mu_{\lambda} \equiv L_{\lambda, \min } / \overline{L_{\lambda}}$. Both lower and upper limits can be made more stringent by considering the whole wavelength information available. We can thus define $\mathcal{N}_{\text {min }}$ by using the maximum value of the lower limit above, and conversely for $\mathcal{N}_{\text {max }}$. This range of allowed $\mathcal{N}$ translates into corresponding ranges for $\nu, E_{\lambda}$ and $C_{\lambda}$. Exactly how stringent $N_{\min }$ is depends on how close $L_{\lambda \text {,min }}$ gets to $C_{\lambda}$. As the chances of the light curve reaching the background level decrease with the increasing superposition of events, one expects the $N_{\text {min }}$ limit to get progressively less stringent as $\mathcal{N}$ increases. The quadratic dependence on the observed quantities also conspires to broaden the range of $\mathcal{N}$.

This very general and straightforward formalism can be applied to several variability data sets. In the next section, we apply it to one of the best sets of quasar optical light curves presently available.

\section{ANALYSIS OF THE WISE OBSERVATORY QUASAR} LIGHT CURVES

\subsection{Description of the data}


Giveon et al. (1999) have presented the results of a long term B and R photometric monitoring of 42 optically selected nearby quasars from the Palomar Green (PG) sample. The observations were collected with the Wise Observatory $1 \mathrm{~m}$ telescope over the 1991 to 1998 period. Spectrophotometric measurements at Wise and Steward Observatories were used to complement the light curves of 13 of the objects. Previous results on this and related monitoring campaigns have been published in Maoz et al. (1994), Netzer et al. (1996), and a spectroscopic study of a subsample of the objects has been recently concluded (Kaspi et al. 2000). The reader is referred to G99 for details of the observations. The following quantities are medians over the sample: $n_{\text {obs }}=32$ observations per object, restframe sampling interval of 33 days, rest-frame light curve span of 5.9 years, photometric uncertainty $=0.015 \mathrm{mag}$ (in B), $z=0.16 ; M_{B}=-23.3$.

The apparent magnitude light curves were converted to monochromatic luminosities $L_{\lambda}$ at 4400 (B) and 6400 $\AA$ (R) using the same cosmological parameters as G99 $\left(H_{0}=70 \mathrm{~km} \mathrm{~s}^{-1} \mathrm{Mpc}^{-1}, q_{0}=0.2, \Lambda=0\right)$. The Kcorrection was also performed as in G99, assuming a power-law spectrum, whose slope is defined by the median $\mathrm{B}-\mathrm{R}$ color. This was a minor correction because of the low redshifts in this sample. Galactic extinction corrections were made with the values of $A_{B}$ extracted from $\mathrm{NED}^{3}$, and the extinction law of Cardelli, Clayton \& Mathis (1989, with $R_{V}=3.1$ ), but were mostly negligible $\left(A_{B} \leq 0.17\right.$, median $\left.=0.03\right)$ because of the high galactic latitude of the objects (Schmidt \& Green 1983). Emission lines contribute $\sim 10 \%$ to the $\mathrm{B}$ and $\mathrm{R}$ band luminosities of PG quasars (G99); their effect upon the present analysis is also negligible.

The analysis presented below was carried out with the resulting $L_{B}(t)$ and $L_{R}(t)$ light curves. We note that the estimates of $\mathcal{N}, \nu, \tau$ and the background fractional contribution are cosmology, extinction and K-correction independent. Only the absolute values of $E_{\lambda}$ and $C_{\lambda}$ depend on such factors.

\subsection{Structure Function Analysis: Estimating time-scales and the asymptotic variance}

The most commonly employed tool to extract information on the variability time-scales out of quasar light curves is the Structure Function (Simonetti, Cordes \& Heeschen 1985; Hook et al. 1994; Cristiani et al. 1996). The SF is defined by the mean of $[L(t+\Delta t)-L(t)]^{2}$ over a light curve. For a Poissonian sequence of flares the SF is known to be simply proportional to the $\mathrm{SF}$ of a single isolated flare (Appendix B), and therefore only has structure for time-scales shorter than the flare duration. For large $\Delta t$ the SF converges to twice the intrinsic variance of the process: $S F_{\lambda}(\Delta t) \rightarrow 2 \sigma_{\lambda}^{2}=2 E_{\lambda}^{2} \nu / \tau$ (eq. [7]). This happens because variations on such long time-scales effectively correspond to independent samples of the $L_{\lambda}(t)$ process, as the light curve loses memory of its past.

The SF provides estimates for both the variability time scale and amplitude. We use the notation $\sigma_{\lambda, S F}^{2}$ to distinguish the asymptotic variance derived from the $\mathrm{SF}$ from that computed directly from the light curve $\sigma_{\lambda}^{2}=$
$\overline{L_{\lambda}^{2}}-{\overline{L_{\lambda}}}^{2}-\epsilon_{\lambda}^{2}$ (where the last term corrects for the small effects of photometric error), which may be somewhat underestimated for light curves not much longer than the correlation time-scale since they do not sample the whole power contained in the fluctuation power spectrum. Likewise, $\delta_{\lambda, S F} \equiv \sigma_{\lambda, S F} / \overline{L_{\lambda}}$ denotes the long term net variability.

The SFs were fit with theoretical functions corresponding to different flare evolution models. In all fits we are interested in just two quantities: The flare life-time $\tau$, defined by (5), and $\sigma_{\lambda, \mathrm{SF}}^{2}$. Four models for $l(t)$ were explored: (1) square; (2) exponential; (3) symmetric triangle and (4) asymmetric triangle. Equations and plots for the corresponding SFs are displayed in Appendix B. These are clearly toy-models for the radiative evolution of physical flares, but as shown in Appendix B (Fig. B10) the SF is not very sensitive to $l(t)$. While it is unfortunate that little information about $l(t)$ can be retrieved with this technique, this does make the estimates of $\tau$ and $\sigma_{\lambda, \mathrm{SF}}^{2}$ more robust. This is confirmed by the fits, which showed that all $l(t)$ models produce very similar parameters (with the exception of exponential flares, which in some cases do not converge and are generally poorer). For this reason, we present only the results for square flares.

Let $L_{i}(i=1, \ldots N)$ be the luminosity of a quasar at an epoch $t_{i}$ in a given spectral band. The SF of this light curve may be defined as follows. If $t_{i}$ and $t_{j}$ are two distinct epochs of the light curve, and $t_{i}>t_{j}$, then

$$
s_{i j}=s\left(\Delta t_{i j}\right)=\left(L_{i}-L_{j}\right)^{2}-\left(\epsilon_{i}+\epsilon_{j}\right)^{2}
$$

where $\Delta t_{i j}=\left(t_{i}-t_{j}\right) /(1+z)$ is the interval between these two epochs in the quasar rest-frame. In this expression, the observational errors $\epsilon$ are subtracted in quadrature from the luminosity difference in order to "remove" the observational noise from the SF. The data to be fitted, hence, are the set of pairs $\left(\Delta t_{i j}, s_{i j}\right)$. The SF parameters were estimated by direct minimization of the sum of the square residuals between data and model. Note that no binning was applied to data before the fitting. All but the fourth model in Appendix B have two parameters: a time scale $\tau$ and the asymptotic limit $S F(\infty)=2 \sigma_{\lambda, S F}^{2}$ (the fourth model in Appendix B has two time-scales). The errors in the parameters were obtained by bootstrap. In this technique an observed distribution with $n_{\text {obs }}$ data points is resampled many times, each time picking a random set of $n_{\text {obs }}$ data points (allowing for repetition) out of the original ones. The underlying hypothesis is that the actually observed values trace the distribution of the measured quantities. For each resampled data set the parameters are fitted, and the parameter errors are estimated from the variance of the values estimated in all resampled data sets. Initially the B and R SFs were fitted separately. A global estimate of $\tau$ combining the $\mathrm{B}$ and $\mathrm{R}$ data was then performed, fitting both SFs with the same value of $\tau$ but different $\sigma_{B, S F}^{2}$ and $\sigma_{R, S F}^{2}$.

$\mathrm{B}$ and $\mathrm{R}$ SFs for five objects are illustrated in Fig. 1 along with their individual and global fits. The SFs in this figure were computed binning the squared luminosity differences in $\Delta t$ such that each bin contained 25 points.

\footnotetext{
${ }^{3}$ The NASA/IPAC Extragalactic Database (NED) is operated by the Jet Propulsion Laboratory, California Institute of Technology, under contract with the National Aeronautics and Space Administration.
} 


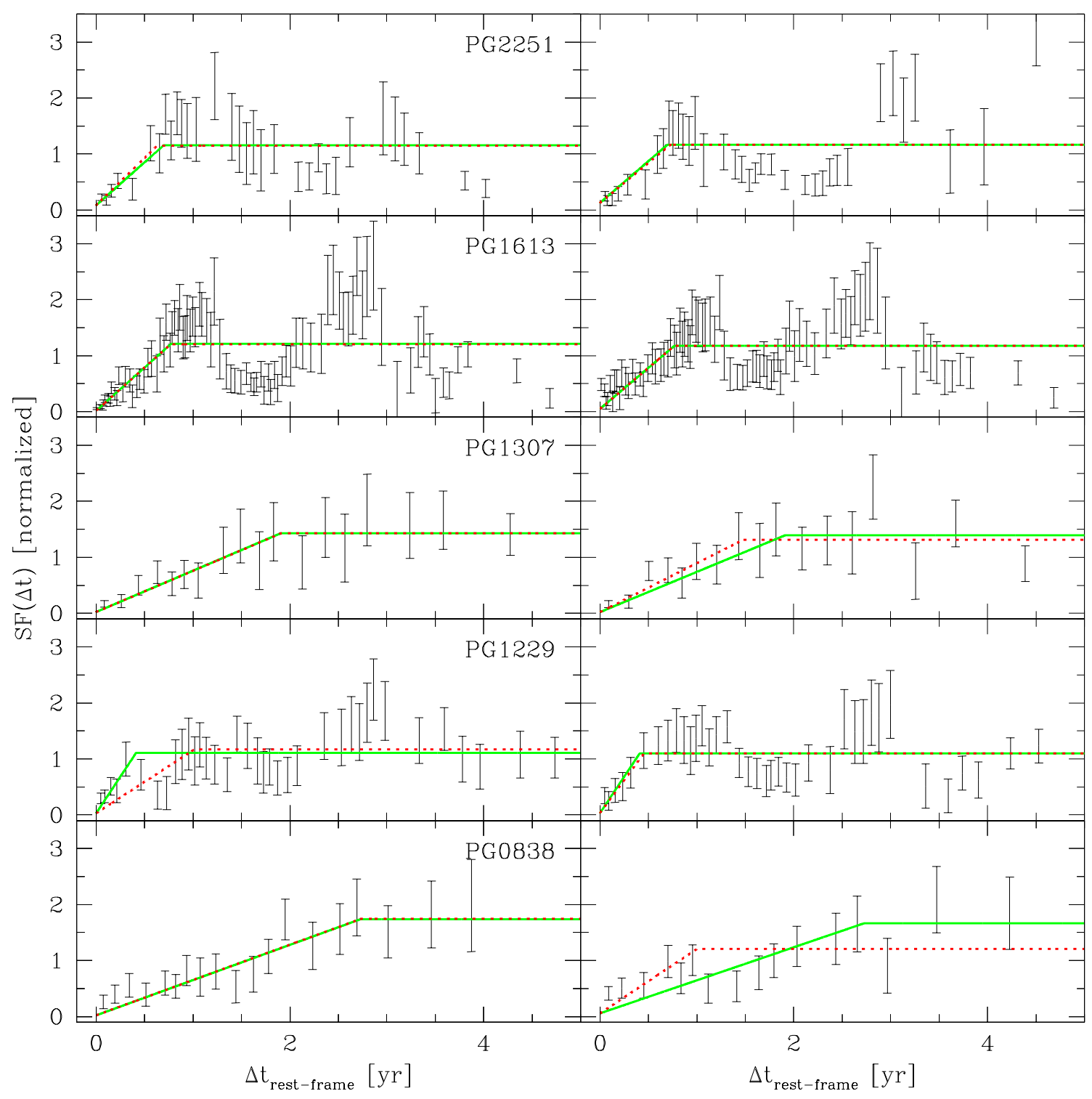

Fig. 1.- Illustration of the Structure Functions and their fits for 5 quasars. Left and right panels show the results for B and R bands respectively. Dotted lines correspond to the individual SF fits, while solid lines indicate the SF resulting from the combined $\mathrm{B}$ and $\mathrm{R}$ fits. The $(L(t+\Delta t)-L(t))^{2}$ differences were grouped in bins of 25 points and the error bars in the SF were computed with the bootstrap method. This was done for plotting purposes only, since the fits do not involve binning the SF. All fits shown correspond to square flares. The SFs are normalized to twice the observed light curve variance $\left(2 \sigma_{\lambda}^{2}\right)$ for convenience.

This was done just for plotting purposes, as the fitting procedure does not involve binning. The error bars in Fig. 1 were computed bootstrapping the light curves 1000 times.

Though the fits were satisfactory for most objects (e.g., PG 0838 and 1307 in Fig. 1), very often the SFs exhibit complex shapes with ups and downs (e.g., PG 1613 and 2251). Such a non monotonic behavior does not rule out the hypothesis that the light curve is made up by the Poissonian superposition of flares with simple profiles, as large departures from the predicted SF also occur for simulated light curves when these are sampled a finite number of times, as will be explained in Fig. 4 and §3.4.1. Theoretical SFs are derived under the assumption of infinitely long light curves, and it would be naive to expect observed SFs to exhibit the smooth, well behaved shapes predicted by theory. This point has to be kept in mind when evaluating the fits performed in this section. One must therefore exert caution when interpreting the fit parameters, particularly $\tau$.

Results of the fits are presented for all 42 sources in Table 1, along with other useful light curve statistics. Though in many cases the life-times inferred from both wavelengths agreed well, we find a large scatter around the $\tau_{B}=\tau_{R}$ line (see also G99). For the reasons discussed above, we believe this is more likely an artifact of the finite span of the light curves than a real effect, and in what 
follows we shall only refer to the values of $\tau$ obtained with the combined $\mathrm{B}$ and $\mathrm{R}$ fits. The asymptotic net standard deviations $\delta_{B, S F}$ and $\delta_{R, S F}$ in Table 1 also correspond to those obtained in the combined fits, but we remark that these were very similar for the individual and global fits.

For four objects, PG 0026, 1100, 1427 and 1626, we find that the SF does not converge over the time span of the observations. All these quasars present rising or decreasing trends over all the length of the observations (Fig. 2 of G99). This suggests either long ( $\gtrsim 5 \mathrm{yr}$ ) time scales, an underlying slowly varying component, or else may be indicative of a non-statistically stationary process. In addition, for PG 1114 and 1512 we find time-scales close to the length of the light curve. These six objects were discarded from further analysis.

Fig. 2 shows how the net variability measured directly from the light curve compares with the SF-determined long term net variability. One finds that $\delta_{\lambda, S F}$ is some $17 \%$ larger than $\delta_{\lambda}$ for both $\mathrm{B}$ and $\mathrm{R}$, confirming that the latter slightly underestimates the long term variability. Also shown in Fig. 2c is a comparison of the net variabilities in the $\mathrm{B}$ and $\mathrm{R}$ bands, to illustrate the fact that $\delta_{B, S F}>\delta_{R, S F}$ by typically $\sim 30 \%$.

The diversity of time-scales obtained in this analysis (from $\sim 0.5$ to $3 \mathrm{yr}$ ) is consistent with the results of G99, who found a wide range of zero-crossing auto-correlation time-scales. In fact, our values of $\tau$ are similar to theirs, but with a substantial scatter. In the context of Poissonian models, this diversity reinforces the suspicion $(\S 1)$ that quasar flares are not all the same, and that the $\delta \propto \bar{L}^{-1 / 2}$ may not be universal. Indeed, in this more general scenario, the "generalized Poissonian model" of Cid Fernandes et al. (1996), every quasar lies in one of a family of $\delta \propto \bar{L}^{-1 / 2}$ laws (see Fig. 6). Members of each such family have different rates, but same energy, life-time and background contribution (eqs. [6] and [7]). Also, this intrinsic diversity casts doubts upon the meaning and usefulness of ensemble SFs.

\subsection{Constraints on the energies, rate and background contribution}

The asymptotic net variabilities $\delta_{B, S F}$ and $\delta_{R, S F}$ obtained above, along with the values of $\bar{L}_{\lambda}$ and $\mu_{\lambda}$ (listed in Table 1), can be directly applied on eq. (11) to constrain the allowed range of $\mathcal{N}, c_{B}$ and $c_{R}$. We remark that this is nearly independent of the SF analysis, since $\delta_{\lambda, S F}$ is not too different from $\delta_{\lambda}$ (Fig. 2) and $\tau$ is not employed in these constraints. The results of this calculation are presented in Table 2 .

Except for 3 out of 36 quasars, the upper limit $\mathcal{N}_{\max }$ is always given by the $\mathrm{B}$ band, since, as already noted by G99, the variations there are larger than in R (Fig. 2c), in agreement with the general tendency of AGN. As anticipated $(\S 2)$, the ranges of the obtained $\mathcal{N}_{\mathrm{s}}$ are wide (factor of $\sim 20)$. Still, they provide useful estimates of a physically meaningful quantity in Poissonian models. From Table 2 one concludes that the typical number of living events in the G99 quasars is of order 5-100.

A corollary of $\mathcal{N}_{\max }$ being defined by the B light curves is that non trivial lower limits for the background component can only be obtained for the $\mathrm{R}$ band for the major- ity of objects (Table 2). In some cases, like for PG 1309 and 1354, one finds lower limits of as much as $40 \%$ in R, indicating a substantial contribution from an underlying constant spectral component. The upper limits for $c_{R}$ are given by $\mu_{R}$, tabulated in Table 1 , which for these two quasars are 90 and $86 \%$ respectively.

\subsubsection{Energies and rates}

The $\mathcal{N}$ and $c_{\lambda}$ limits above make no explicit use of the life-times. Estimates for the allowed range of flare rates and energies require knowledge of $\tau$. These are listed in Table 2 for the life-times found in $\S 3.2$. Rates between 1 and a few hundred $\mathrm{yr}^{-1}$, and monochromatic energies from $3 \times 10^{45}$ to $10^{49} \mathrm{erg} \AA^{-1}$ are found for $E_{B}$ and $E_{R}$. Note that the energies for individual quasars are more constrained (typically to within a factor of 4) than either $\nu$ or $\mathcal{N}$, since they only depend on the square root of $\nu$. Furthermore, inspection of Table 2 shows that there is not a single value of either $E_{B}$ or $E_{R}$ that is simultaneously compatible with all lower and upper limits for the G99 quasars. This, again, points to a diversity of flare properties in quasars.

\subsection{Light curve simulations}

The estimates of the basic Poissonian parameters presented here rely on just the mean and minimum luminosities plus the SF-based estimates of the flare life-time and asymptotic variance, this latter quantity being little different from the light curve variance. The actual light curves contain much more information, as they are defined by a particular realization of birth-dates of the flares and their detailed radiative evolution. Retrieving this information from these observations is however an impossible task. We have experimented using higher moments (skewness and kurtosis) of the light curves as further constraints, but this proved fruitless in practice (Appendix A).

Since our estimates are based on so little information, there is no guarantee that a superposition of flares with the inferred properties would bear any morphological resemblance to the observed light curves. In order to verify whether Poissonian models within the bounds defined in $§ 3.3$ can produce quasar-looking light curves we have performed a series of Monte Carlo light curve simulations. Our goal here is to broadly assess the "morphological compatibility" of models and data in a qualitative way, based on a simple visual inspection. The simulations make use of the value of $\tau$ listed in Table 1 , and vary $\nu$ within its empirically defined limits for each quasar (Table 2). The flare energy and background luminosity are determined self consistently through eqs. (9) and (10). Square shaped flares were used for consistency with the results already presented.

In Fig. 3 we present three illustrative examples of this experiment. The top curves in each panel show the B band light curves for PG 0052, 1309 and 1404, as observed in the Wise Observatory campaign. The thin solid line in the bottom illustrates a randomly chosen realization of the light curve, computed with $\nu$ set to half-way between $\nu_{\min }$ and $\nu_{\max }$. The thick solid line shows the simulation which, among thousands of runs, best matches the observed light curve in a $\chi^{2}$ sense, whereas the dashed curve 

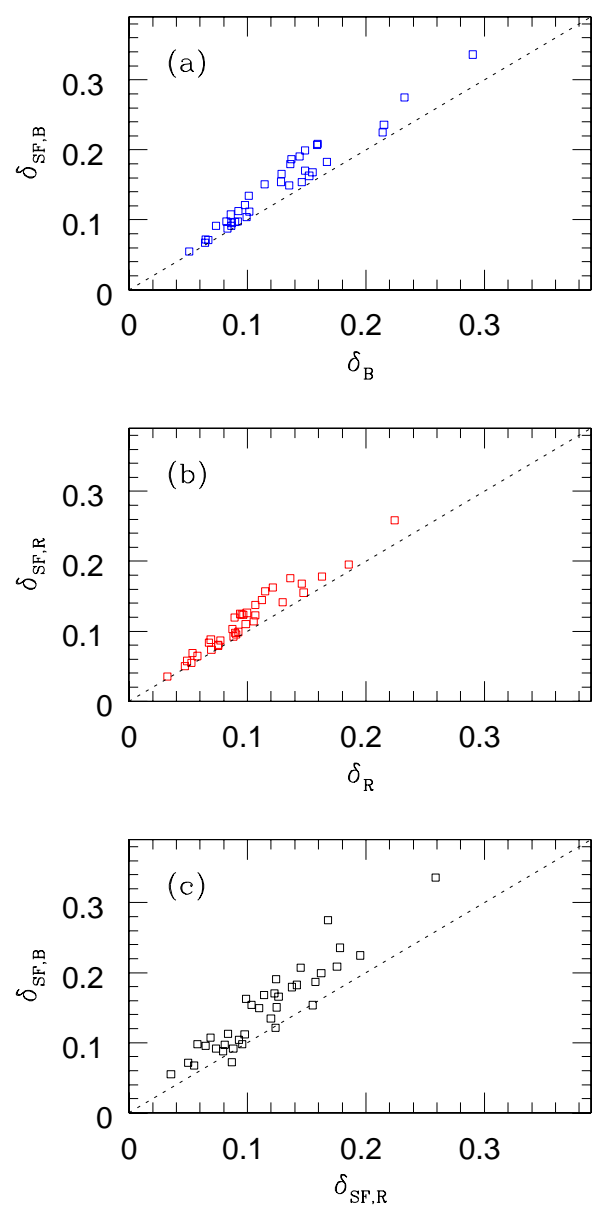

FIG. 2.- Relation between the net variability indices computed from the light curve, $\delta=\sigma / \bar{L}$, and those computed with the asymptotic variance inferred from the SF. (a) B-band. (b) R band. (c) Comparison of B and R band net variability.

shows the same simulation after sampling it with the same rest-frame pattern as the data and with Gaussian perturbations added mimicking the sequence of photometric errors reported in G99. All model light curves were vertically displaced for clarity. By construction, their mean luminosity is essentially identical to the observed one, as are their variances.

Considering the simplicity of the model and the requirement of consistency with the constraints defined in $\S 3.3$, the resemblance between observations and the models is very satisfactory. The "best-matches" are particularly striking. Even better matches would be obtained fitting the light curves, i.e., adjusting both the global parameters and the individual flare birth-dates to optimize the residuals. Of course, the meaning and usefulness of such detailed fits would be questionable given the number of parameters involved. For instance, there are anywhere between 36 and 145 flares for the length of the PG0052 light curve, and up to 1316 in PG1404. Indeed, with so many degrees of freedom, it might be impossible to ever disprove such models. Unlike the flare dates, however, one has much less liberty to play with the global parameters, and our estimates of $\tau, \nu, E_{\lambda}$ and $c_{\lambda}$ go a long way towards constraining the simulations to a region of parameter space capable of pro- ducing quasar looking light curves. Simulations with much longer or shorter time scales, for instance, do not resemble the observations at all. We therefore conclude that even a simple Poissonian superposition of square flares is capable of producing light curves which are very similar to quasars and simultaneously compatible with their basic light curve statistics.

\subsubsection{Structure Function}

The SFs of PG 0052 and 1404 are compared to the corresponding simulated light curves in Fig. 4. As anticipated, relatively large deviations from the smooth theoretical curve occur even for model light curves. The bottom panels show how the SF is improved with light curves twice to five times longer than the observed ones. These were constructed simply extending the sampling of the model by patching together 2 (panels e and $\mathrm{f}$ ) or 5 ( $\mathrm{g}$ and $\mathrm{h}$ ) sequences of the actual observing dates for these two quasars, thus maintaining the G99 sampling pattern. Even for such long (13-45 yr) hypothetical light curves the SF oscillates, though one sees it gradually converging to its statistically expected shape and amplitude. Increasing the sampling rate is not as benefic as increasing the length of the data train, as SF oscillations on intervals $\Delta t$ are only averaged 




FIG. 3.- Comparison of observed quasar B-band light curves (top dotted lines) and simulations. Thin solid lines show a randomly chosen simulation. The thick lines indicate a "best match" model (see text), while the dotted line show the same model after sampling as in the data. All light curves are normalized to $\overline{L_{B}}$ (Table 1), and the simulations are shifted downwards for clarity. The global Poissonian parameters for the simulations in thick lines are $\tau=0.70,1.87$ and $0.47 \mathrm{yr} ; \nu=19,47$ and $223 \mathrm{yr}^{-1} ; E_{B}=4.8 \times 10^{47}, 1.8 \times 10^{47}$ and $4.9 \times 10^{45}$ erg $\AA^{-1}$; $c_{B}=15,8$ and $7 \%$ for PG 0052, 1309 and 1404 respectively. The simulations in the bottom thin lines have the same lifetimes as above, but $\nu=16,29$ and $131 \mathrm{yr}^{-1} ; E_{B}=5.2 \times 10^{47}, 2.4 \times 10^{47}$ and $6.3 \times 10^{45} \mathrm{erg} \AA^{-1} ; c_{B}=21,28$ and $29 \%$ for PG 0052,1309 and 1404 respectively. These values are all, by construction, consistent with the limits in Table 2 .

out for $\Delta t \gg \tau$, i.e, as independent (separated by $\sim \tau$ ) portions of the light curve are sampled a statistically significant number of times. This explains why even long light curves exhibit long term SF 'noise' but are much better defined on short time scales (Fig. $4 \mathrm{e}-\mathrm{h}$ ).

These experiments illustrate the inherent difficulty in modeling SFs of stochastic processes. As a further example, we note that the SF analysis of a longer (11 yr) light curve of PG 1226 (= 3C 273) by Paltani, Courvoisier \&
Walter (1988) suggests a value of $\tau$ of $\sim 0.6 \mathrm{yr}$, a factor of 4 smaller than the $2.3 \mathrm{yr}$ we found for the $5.5 \mathrm{yr}$ G99 monitoring of this quasar. The SF of PG 1226 is similar to that of PG 1229 in the B band (Fig. 1), with an initial peak at small $\Delta t(\sim 0.4 \mathrm{yr})$, followed by oscillations on longer time scales. Whereas for PG 1229 the global fits favored the small $\tau$, in PG 1226 the amplitude of the 0.5 yr peak is smaller, and a longer $\tau$ was favored, similar to what is seen in the R band SF of PG 0838 (Fig. 1). Even 


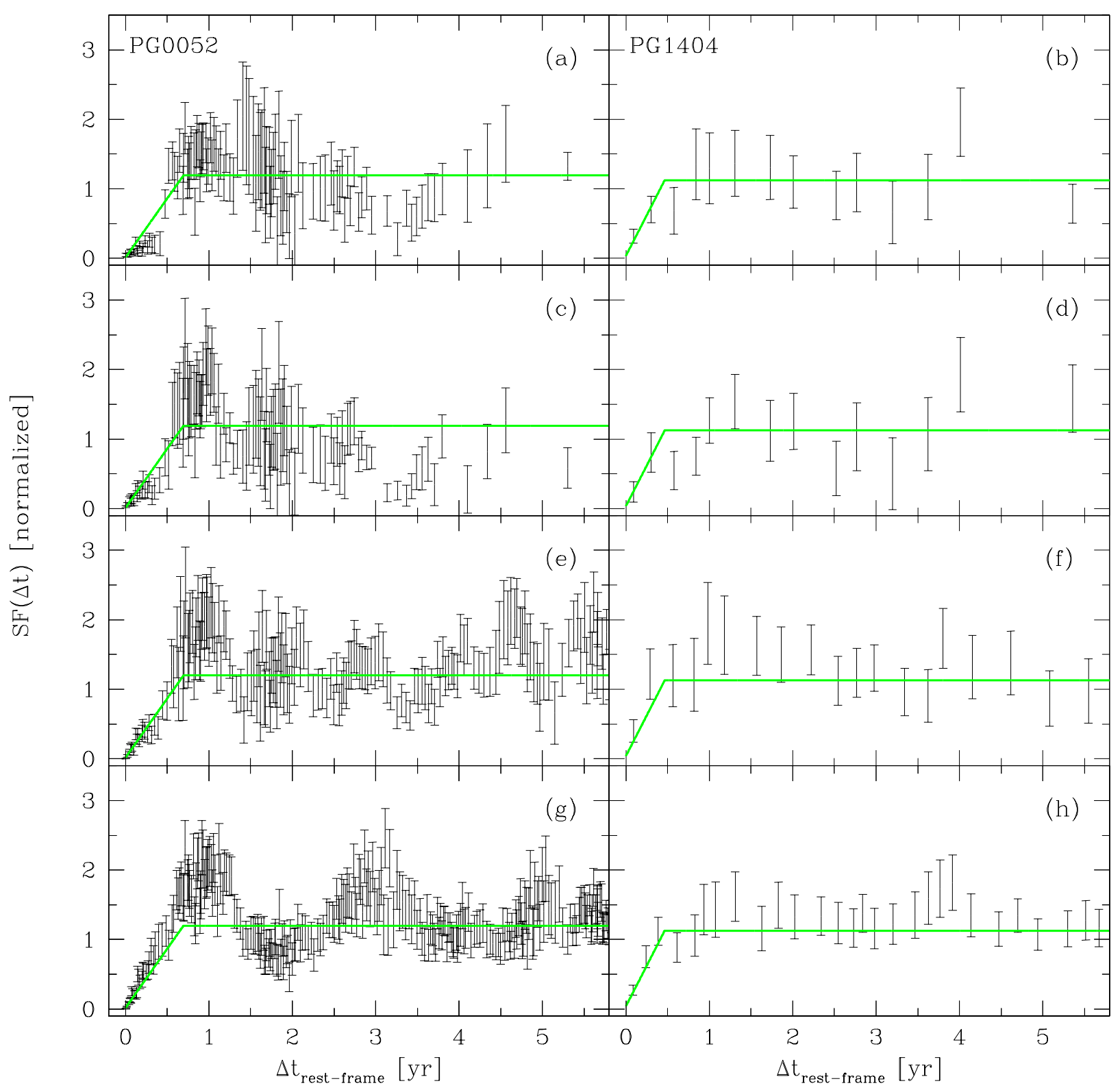

FIG. 4.- Structure Function of PG 0052 (panel a) and PG 1404 (b) in the B band. Panels c and d show the SFs of the corresponding simulated light curves (shown as dashed lines in Fig. 3). Simulations twice and five times longer than the length of the Wise Observatory monitoring of these quasars were used to compute the SFs in panels e and $\mathrm{f}(2 \times)$, and $\mathrm{g}$ and $\mathrm{h}(5 \times)$. In all plots the solid line marks the same combined B and R SF fit performed upon the observed light curves. All SFs are normalized to twice the observed $\sigma_{B}^{2}$.

considering the differences in technicalities of SF analysis between different works, one is forced to conclude that the actual uncertainties in the SF parameters is likely to substantially exceed the formal fit-errors (about $5 \%$ of the parameter values).

Overall, we conclude that SF analysis for individual objects is just starting to become possible, despite the excellent quality of the G99 light curves. We are however confident that the fits performed in this paper are correct to within better than an order of magnitude, and thoroughly meet our goal of providing rough but useful estimates for the basic parameters of Poissonian models for the optical variability of quasars.

\section{DISCUSSION}

Poissonian models provide a simple and elegant mathematical framework which relates basic parameters to measurable quantities, as demonstrated by the results presented above. The same technique can be applied to other data sets and spectral bands, thus increasing the number of constraints. In particular, it will be interesting to apply it to the spectrophotometric monitoring observations of 
28 quasars, all in the G99 sample, reported in Kaspi et al. (2000). This should yield a clearer picture of the (optical) spectral energy distributions of the variable and constant components, as well as better constrained parameters than was possible with only two wavelengths. An illustration of how important it is to properly define $\overline{L_{\lambda}}$ and $\delta_{\lambda}$ is given in Fig. 5.

The $E_{B}$ limits listed in Table 2 encompass both the estimates of Cid Fernandes etal. (1996) and Garcia et al. (1999) for different samples. Their estimates, however, were based on the properties of the ensemble of quasars, and assumed typical variability time-scales, whereas here we attempted to treat each quasar individually. In fact, this is the first time that constraints on Poissonian parameters are computed on an object by object basis for a large number of quasars.

A further comparison can be made with the results of Paltani etal. (1988) for 3C273. We estimate from their plots a monochromatic flare energy of $\sim 2 \times 10^{47} \mathrm{erg} \AA^{-1}$, about an order of magnitude smaller than $E_{B, \min }$ in Table 2. This discrepancy is explained by the already mentioned $\sim 5$ times smaller $\tau$ found by those authors, as well as their $\sim 2$ times smaller asymptotic net variability compared to the $\delta_{S F, B}=0.13$ in Table 2, and another factor of $\sim 1.5$ due to the redshift effect (see below). The discrepancy in the asymptotic SF variance is mostly due to the fact that they allow for a slowly ( $\gtrsim 10 \mathrm{yr}$ ) varying component, attributed to a blazar behavior. Two of the other 6 radio loud objects in G99 sample (PG 1100 and 1512) show evidence for long time-scale variations which could be due to a similar slow component (marginal evidence that the radio loud objects in this sample present longer time-scales can be seen in Fig. 6c). It is in fact adequate to recall that AGN variability has often been described in terms of the superposition of rapid and slowly varying components (e.g., Lyutyi 1977, Pica et al. 1988). A caveat in the present analysis is thus that what we have been calling a "constant component" may well correspond to an underlying slow process.

We note that no attempt was made to correct any of the variability indices nor the inferred parameters for the variability- $\lambda$ effect which plague fixed band photometric light curves of quasars, leading to overestimated variability amplitudes (Cristiani et al. 1996; Cid Fernandes et al. 1996; Aretxaga etal. 1997; Garcia etal. 1999). Fortunately, the low redshifts of the G99 quasars minimize this effect. For the median $z$ of the sample, and using the parameterization of the $\delta \times \lambda$ relation of Garcia et al. (1999), we estimate that the variability indices in Table 1 (columns 4 to 7 ) would need to be multiplied by typically 0.8 to obtain the rest-frame indices. This would increase $\mathcal{N}_{\text {min }}$, $\mathcal{N}_{\text {max }}$ and the corresponding $\nu$ limits by some $56 \%$ while reducing the energies by a factor of 0.64 . These are relatively small factors considering the allowed ranges for the parameters and were not applied in Table 2. Furthermore, such corrections would soon become obsolete since the spectral data collected by Kaspi et al. (2000) allows the direct computation of rest-frame continuum variability indices. We did, however, experiment with the $\lambda$-correction in the correlation analysis presented next.

\subsection{Correlation Analysis}

Perhaps the most puzzling result of G99 was the finding that there are few convincing correlations between variability indices and a large array of other observed properties for the PG quasars. In Table 3 we synthesize the results of a correlation analysis analogous to that performed by G99, but for our SF-based variability indices. The table is similar to their Table 4, from which the multiwavelength data was borrowed, and lists the percentage probabilities $\left(P_{r}\right)$ of no correlation in a Spearman's rank test, small values indicating significant correlations and negative values corresponding to anticorrelations. Entries between parentheses correspond to correlations after correction of the variability amplitudes with the $\delta-\lambda-z$ relation following Garcia et al. (1999).

The anti-correlation between the variability amplitude (here measured by $\delta_{S F, B}$ and $\delta_{S F, R}$ ) and the optical luminosity found in other studies (e.g., Hook et al. 1994) is essentially absent for this sample, as illustrated in Fig. 6a. Somewhat more significant anti-correlations are obtained by applying the $\lambda$ correction (Fig. 6b; Table 3), but in both cases the correlations here are even weaker than those found by G99. The dotted lines in Fig. 6a and b mark the prediction of simple Poissonian models in which $c_{\lambda}, E_{\lambda}$ and $\tau$ are identical for all quasars. This illustrates the often claimed failure of this model (e.g. Hook et al. 1994), and the necessity to allow for a diversity of values for these parameters to account for the observed variability properties in the framework of Poissonian processes.

The variability time-scale seems to increase with luminosity (Fig. 6c), but, as in G99, with a large scatter. Previous evidence for such a correlation was reported by Cristiani etal. (1996), in an analysis of the ensemble SF of 486 objects spanning a much larger range of luminosities and redshifts. It is interesting to note that applying their Model E SF-fits to the typical absolute magnitudes of G99 quasars yield $\tau$ between $\sim 0.5$ and $2.5 \mathrm{yr}$, compatible with our individual SF fits. We also find $\tau$ to correlate positively with the $\delta_{S F}$ 's, but this may be due to a trade-off effect in the SF fitting (\$3.4.1). The weak anticorrelation with $\alpha_{o x}$ found by G99 is much stronger here (Fig. 6d).

The good correlations between the variability amplitude and the equivalent widths of $\mathrm{H} \beta$ and HeII $\lambda 4686$ found by G99 are reproduced at about the same high level of significance (Fig. 7a). Unlike G99, however, we find the optical to X-ray index $\alpha_{o x}$ to be significantly anticorrelated with both $\delta_{S F, B}$ (Fig. 7d) and $\delta_{S F, R}$. All these correlations are improved with the $\lambda$-correction.

A correlation was found between the ratio of the $B$ to $R$ asymptotic standard deviations and $\overline{L_{B}}$ as well as $z$. This could be due to the redshift effects already discussed, but in this case a $\delta$ - $\lambda$ law different from that of Garcia et al. (1999) would be implied, since the $z$-effects upon $\sigma_{B, \mathrm{SF}} / \sigma_{R, \mathrm{SF}}$ cancels out in their parameterization. Alternatively, if this interpretation is proved wrong, one would conclude that the flares are bluer in more luminous sources (eq. 9).

Overall, we largely confirmed G99 results, with a few minor differences (e.g., Fig. 6d) undoubtedly due to the different methodologies employed in the definition of variability amplitudes and time-scales. Despite the few good correlations found, whose meaning we discuss below, a 


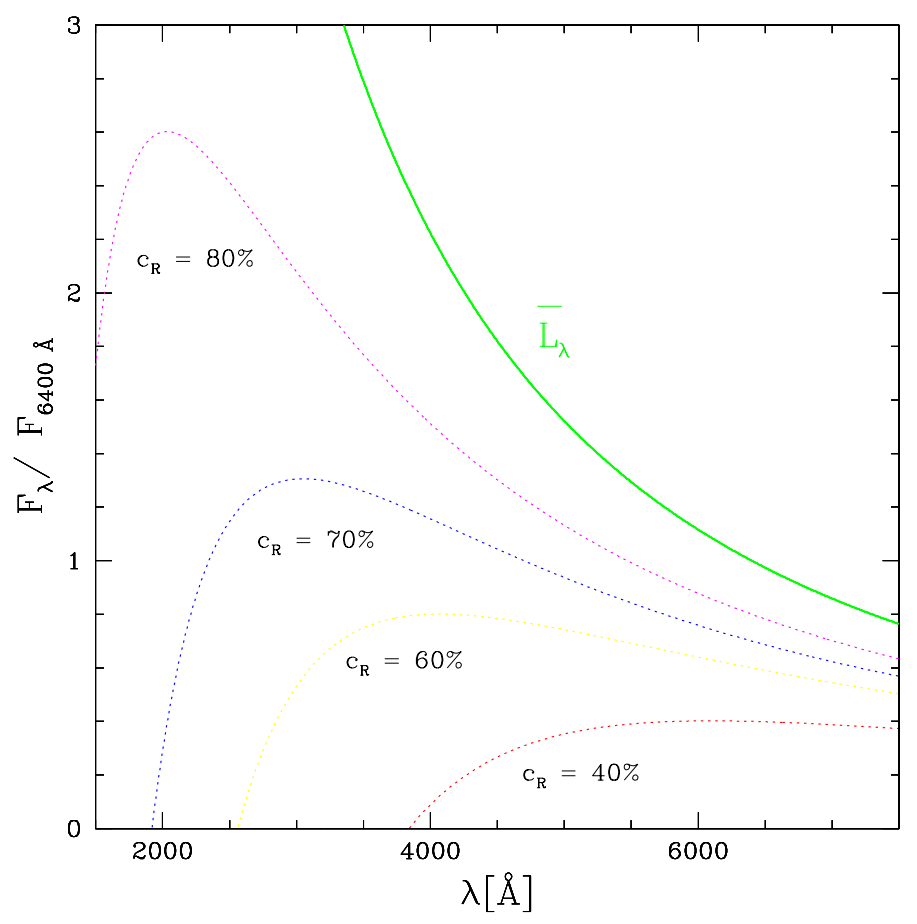

FIG. 5.- Background components $\left(C_{\lambda}\right.$, dotted lines) for a hypothetical quasar with $\overline{L_{\lambda}} \propto \lambda^{-1.7}$ (top solid line) and $\delta_{\lambda} \propto \lambda^{-1}$. The plot illustrates how the spectral energy distribution of the constant component can be deduced from (observationally) well determined $\overline{L_{\lambda}}$ and $\delta_{\lambda}$, coupled with variability derived constraints upon $c_{\lambda}$ at any particular wavelength.

striking result of this analysis is the large scatter present even in the best correlations obtained. In any scenario for AGN, the variability properties must somehow be dictated by the physical conditions prevalent in the active nucleus (accretion rate, black hole mass, orientation, etc.). Since these conditions must also define other aspects of AGN phenomenology, it is natural to expect that properties driven by common causes should exhibit some degree of correlation. Given the controversial history of correlations in quasar variability studies, however, it is perhaps not so surprising that so few good correlation were found.

Regarding Poissonian models, one would obviously like to search for correlations between the basic parameters and other properties. The constraints upon $\mathcal{N}, \nu, E_{\lambda}$ and $c_{\lambda}$ derived in this paper are however not strong enough to warrant a proper correlation analysis. Taking the middle value between $\mathcal{N}_{\min }$ and $\mathcal{N}_{\max }$ as a measure of $\mathcal{N}$, we obtain an anticorrelation between $\mathcal{N}$ and $\mathrm{EW}(\mathrm{H} \beta)$ and a positive correlation with $\alpha_{o x}$, both with $P_{r}$ at the $1 \%$ level. The flare rate follows the same trends. For the reason discussed above, it would be premature to give much emphasis to these correlations.

\subsubsection{Interpretation of the correlations with $E W(H \beta)$}

A simple interpretation of the significant correlation between $\operatorname{EW}(\mathrm{H} \beta)$ and the variability amplitude is possible by postulating that the variable component dominates the ionization of the gas. This idea is also compatible with our earlier conclusion $(\S 2)$ that the variability-wavelength anticorrelation indicates the dilution of a blue variable component by a red underlying background. In this hypothesis both variable $\left(V_{\lambda}\right)$ and constant $\left(C_{\lambda}\right)$ components con- tribute to the continuum under $\mathrm{H} \beta$, but only the former is proportional to the ionizing luminosity $\left(L_{i o n}\right)$, so one predicts

$$
E W(H \beta) \propto \frac{\nu E_{B}}{\nu E_{B}+C_{B}}
$$

where we used the $\mathrm{B}$ band because of its proximity to $\mathrm{H} \beta$. This relation may be rewritten as

$$
E W(H \beta) \propto \mathcal{N}^{1 / 2} \delta_{S F, B}=v_{B}=1-c_{B}
$$

which reveals a proportionality between $\operatorname{EW}(\mathrm{H} \beta)$ and $\delta_{S F, B}$. Obviously, the same prediction applies to $\mathrm{EW}(\mathrm{HeII})$.

Using $\mathcal{N}=\left(\mathcal{N}_{\text {min }}+\mathcal{N}_{\text {max }}\right) / 2$, the product $\mathcal{N}^{1 / 2} \delta_{S F, B}$ correlates at the $P_{r}=0.6 \%$ level with $\mathrm{EW}(\mathrm{H} \beta)$, in agreement with the prediction. Since our estimates of $\mathcal{N}$ are not independent of $\delta_{S F, B}$ (eq. 11), it is perhaps more meaningful to test the predicted relation using $c_{B}$, for which we have a robust upper limit imposed by the minimum in the light curve $\left(\mu_{B}\right.$ in Table 1$)$. In Fig. $7 \mathrm{~b}$ we see that the expected anticorrelation is confirmed with a high significance (Table 3). Even considering that $\mu_{B}$ is an upper limit to $c_{B}$ and the scatter in the plot, it is interesting to see that the trend is roughly linear, as predicted.

$\mu_{B}$ is also strongly correlated with $\alpha_{o x}\left(P_{r}=0.1 \%\right)$, the X-ray spectral index $\alpha_{x}\left(P_{r}=1.8 \%\right)$, and anticorrelated with $\mathrm{EW}([\mathrm{OIII}])\left(P_{r}=0.8 \%\right)$, [OIII $]$ to $\mathrm{H} \beta$ peak intensities ratio $\left(P_{r}=0.2 \%\right)$ and $\mathrm{EW}(\mathrm{HeII})\left(P_{r}=0.5 \%\right)$. The correlation with $\alpha_{o x}$, in particular, gives strength to our working hypothesis, insofar as this index can be interpreted as indicative of the ratio between the constant and variable (ionizing) components. 

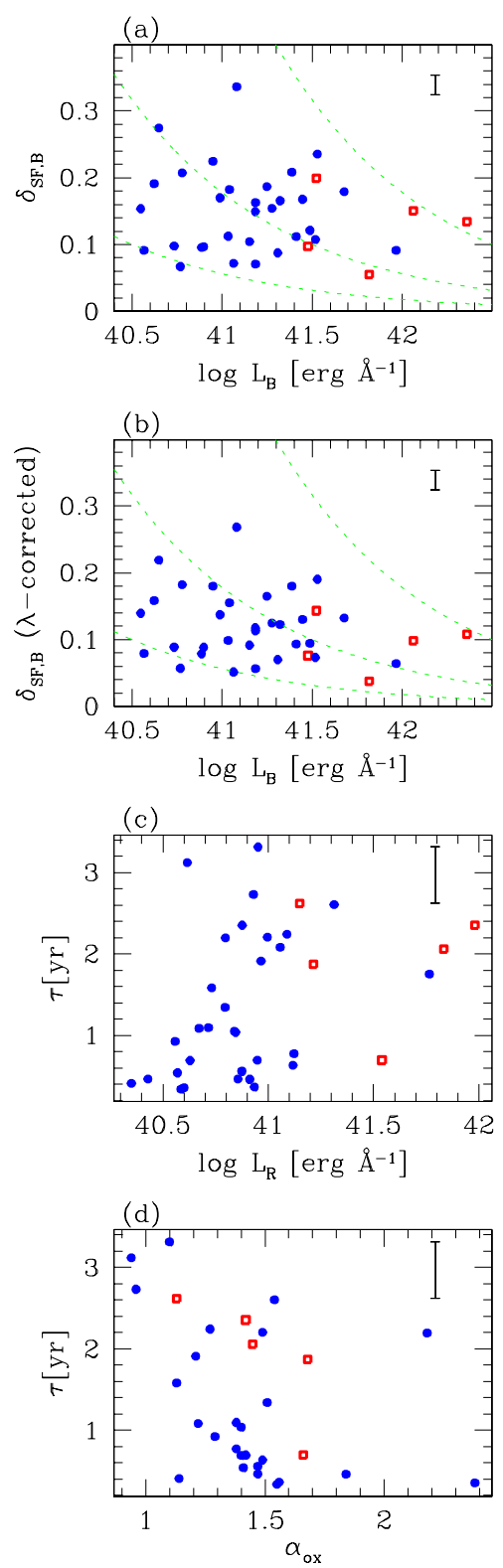

FIG. 6.- Correlations between variability properties and luminosity $(\mathrm{a}-\mathrm{c})$ and $\alpha_{o x}(\mathrm{~d})$. Filled circles mark radio quiet objects, whereas squares correspond to radio loud sources Dotted lines in the top panels are simple Poissonian models with $0.5 \log \left(1-c_{B}\right) E_{B} / \tau=40.5$ (top line), 39.5 (middle) and 38.5 (bottom). The error bars on the top right of each panel represent the mean formal uncertainties in $\delta_{S F}$ and $\tau$ as obtained from the SF fits.

By admitting that different quasars have different background fractions, and that $V_{\lambda}$ dominates the ionization, we naturally explain at least some of the correlations observed. This argues against $C_{\lambda}$ being part of the ionizing source, such as hot but non-flaring portions of the accretion disk surface. Outer, colder regions of the disk can contribute to $C_{\lambda}$, as do the host galaxy starlight and other circum-nuclear sources.

The alternative hypothesis that $L_{i o n}$ is proportional to the total $V_{\lambda}+C_{\lambda}$ components would not induce the correlations above, while the hypothesis that $L_{i o n}$ is governed by the constant component alone can be straightforwardly rejected, since this would not produce emission line variability.

We note that our eq. (13) is formally and philosophically identical to eq. (10) of Aretxaga \& Terlevich (1994), who identify $C_{\lambda}$ with a young nuclear star-cluster and $V_{\lambda}$ with compact SN remnants exploding out of the same cluster (see also Aretxaga et al. 1997). Their analysis of $\mathrm{EW}(\mathrm{H} \beta)$ aimed to understand the near universal value of this quantity across the AGN luminosity scale (Binette, Fosbury \& Parker 1993). In this model, a range of $\mathrm{EW}(\mathrm{H} \beta)$ values 
could be linked to either different SN explosion energies, or, as is more likely, to different $\mathrm{H} \beta$ and continuum production efficiencies in the SN remnants (see Cid Fernandes 1997 for a review of the pros and cons of the starburst model). Stochastic effects could also play a role in defining the $\mathrm{EW}(\mathrm{H} \beta)$ and variability relation, something which can happen in any Poissonian scenario. Indeed, the tentatively identified anticorrelation between $\mathcal{N}$ (and $\nu$ ) and $\operatorname{EW}(\mathrm{H} \beta)$ may be indicative of stochastic effects in the G99 data, and may be partly responsible for the scatter in Fig. 7a and d.

\subsection{Relation to physical models}

The ultimate goal of the Poissonian description of quasar variability is of course to extract information on the fundamental properties of the variability phenomenon and use them to guide and discriminate between physical theories. A detailed discussion of the implications of the estimates presented in $\S 3$ to different scenarios for AGN variability is beyond the scope of the present paper, but we would like to take one particular model as an illustration of the possible associations between the observationally constrained parameters discussed here and physically meaningful quantities.

In the model proposed by Haardt et al. (1994), blobs emerge from the surface of an accretion disk and release magnetically stored energy in the form of rapid X-ray flares. In the X-ray regime, the background fraction in this model would be associated with the fraction of the disk area covered by blobs (see Figure 1 of Galeev, Rosner \& Vaiana 1979), which equals the ratio of charge and discharge times. As they postulate the optical-UV variability to be driven by the $\mathrm{X}$-ray flares, $c_{\lambda}$ is related to the fraction of the reprocessed luminosity and the optical-UV emission from the underlying stable component of the disk, but note that comparison with the limits on $c_{\lambda}$ derived in this paper require allowance for the diluting effects of larger scale sources, such as the host galaxy or an extended scattering region. One of the predictions of this model is that at any time there are of order 10 active blobs, independent of the source luminosity, consistent with the limits on $\mathcal{N}$ established here. Also the reprocessed luminosity, which we would identify with $(1-c) \bar{L}=\nu E$ in our formalism, is predicted to be similar to the X-ray luminosity. It is not clear, however, if the time smearing of the minutes long flares during the disk illumination which results in the reprocessing of the blobs energies can be made compatible with the $\sim 0.5-3$ yr time scales implied by the $\mathrm{SF}$ analysis. Surely this must impose some limits upon the geometry of the reprocessing surface. Furthermore, it remains to be established whether the flare energies can be made compatible with the limits laid out in $\S 3.3$. A more detailed scrutiny of the Haardt et al. (1994) model must therefore await more theoretical developments. On the observational side, a Poissonian analysis of X-ray light curves of quasars (similar to that done by Cid Fernandes 1995 for EXOSAT light curves of Seyferts) could also set constraints upon this particular model.

Despite their generality and appealing aspects, Poissonian models are by no means the only possible description of AGN variability. For instance, Mineshige \& Shields (1990) showed that thermal limit cycles similar to those known to occur in dwarf novae (Warner 1995) can also take place in AGN disks, leading to eruptions followed by quiescent periods. Such a model, in which the degree of variability is "self-regulated", would invalidate the formalism employed here, which fundamentally rests upon the hypothesis of independence of the flares and statistical stationarity. The same applies to scenarios involving random walk-like or other kinds of state dependent behavior (e.g., Begelman \& De Kool 1991; Stern, Svensson \& Sikora 1991) or periodic modulations associated to, for instance, precessing jets (e.g., Abraham \& Romero 1999). Testing the Poissonity of AGN light curves is however a hard task. Fourier phase coherence studies like that performed by Krolik, Done \& Madejski (1993) for X-ray light curves of Seyferts, or intermittency tests (Vio et al. 1992) like the one carried out by Longo et al. (1996) for the historical light curve of NGC 4151, can in principle help discriminating between Poissonian models and scenarios where the variations are due to coherent oscillations of a single entity, but these require more data points than are currently available from quasar optical monitoring studies. Therefore, until proven wrong, the Poissonian description may be regarded as a useful tool to unveil physically meaningful properties of AGN.

\section{SUMMARY AND CONCLUSIONS}

We have reviewed the Poissonian formalism for quasar variability in an attempt to provide a general framework which allows fundamental parameters to be estimated from good monitoring data. This was applied to the 6 yr long B \& R light curves of 42 PG quasars obtained by G99, yielding constraints for the energy, rate and lifetimes of the flares. Our main results can be summarized as follows.

(1) The only reasonable way to account for the fact that quasars vary more at shorter wavelengths within a Poissonian scenario is to include the diluting effects of an underlying "non-variable background" component redder than the spectral energy distribution of the putative flares.

(2) A wide range of flare energies, lifetimes, and/or background fractions has to be invoked to account for the observed variability properties (amplitudes and time-scales). This "stretching" of the simplest Poissonian scenario (in which all parameters are the same for every quasar) is warranted by the model independent result that quasars present a wide range of variability time scales, from $\sim 0.5$ to more than $3 \mathrm{yr}$, as inferred from a Structure Function analysis.

(3) The mean number of living flares is constrained to be of order $\mathcal{N} \sim 5$ to 100 , and lower limits for the Rband background contribution of typically $25 \%$ are established. These estimates are independent of cosmology, Kcorrection and extinction, and little sensitive to the SF analysis.

(4) Flare rates between $\sim 1-100 \mathrm{yr}^{-1}$ and monochromatic flare energies in the $\sim 10^{46-48} \operatorname{erg} \AA^{-1}$ range are implied by the data. Overall, the Poissonian parameters for individual quasars are constrained to within about an order of magnitude.

(5) Light Curve simulations were performed and demonstrate the ability to reproduce the observed morphology of quasars light curves extremely well even for a blatantly simplistic "on/off" square-shaped model for the evolution 

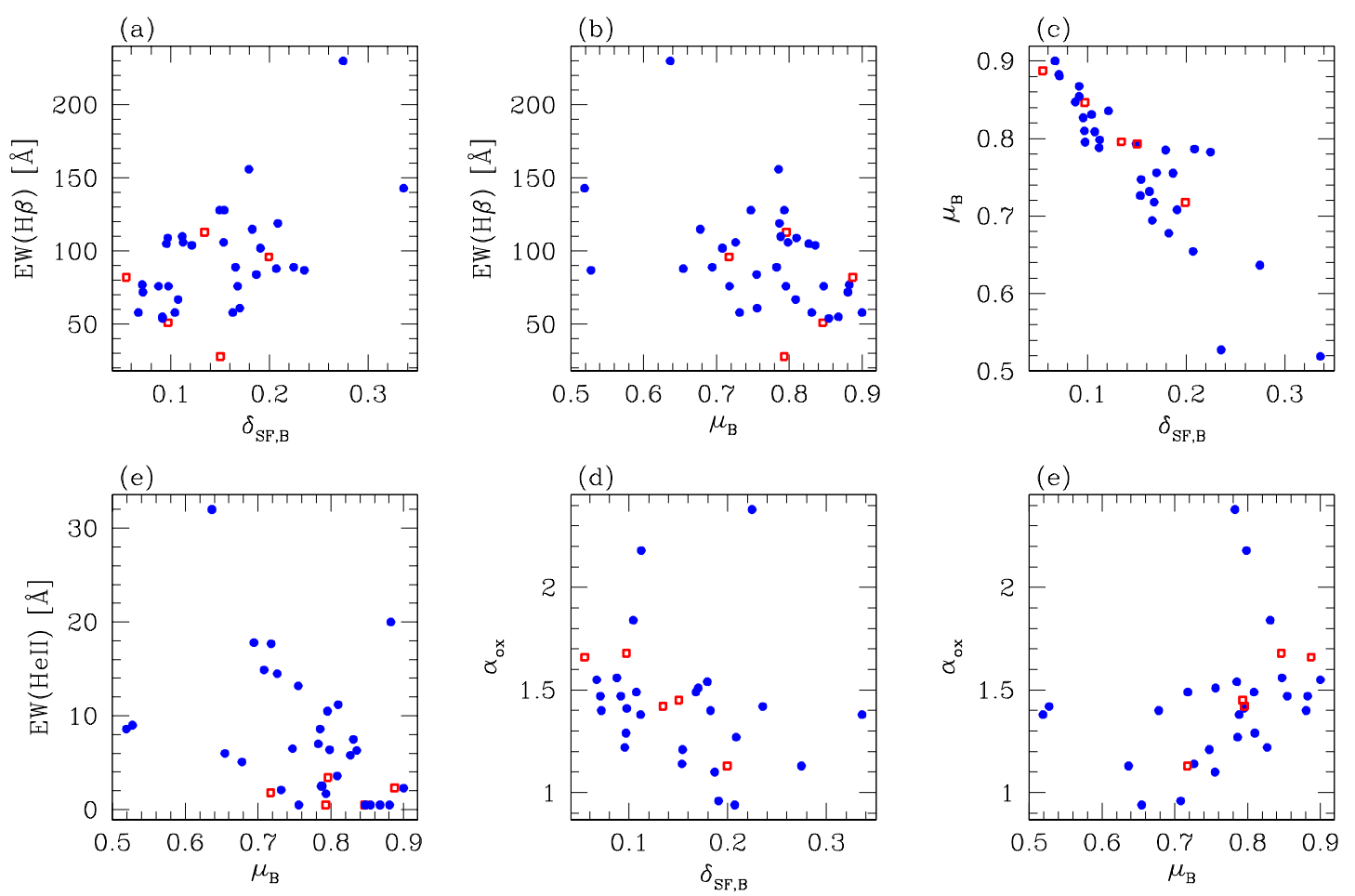

FIG. 7.- Correlations between $\delta_{S F, B}, \mu_{B}, \mathrm{EW}(\mathrm{H} \beta), \mathrm{EW}(\mathrm{HeII})$ and $\alpha_{o x}$ (the larger $\alpha_{o x}$ the softer the spectrum). Note that $\mu_{B}$, the fraction of the minimum to the mean B -band luminosity, is to be seen as an upper limit to true fraction of the background luminosity $\left(c_{B} \leq \mu_{B}\right)$. Symbols as in Fig. 6.

of the individual flares. Indeed, the whole Poissonian analysis is highly insensitive to the flare shape.

(6) Experiments were performed on the use of higher moments of the light curve as further constraints, but found to be of little use at present.

(7) The variability properties of the PG quasars present little correlation with other properties. Even the best correlations identified, like that between the variability amplitude and $\operatorname{EW}(\mathrm{H} \beta)$, present a substantial scatter, confirming the results of G99.

(8) The $\operatorname{EW}(\mathrm{H} \beta) \times$ variability amplitude was interpreted in a scenario where only the variable component participates in the ionization of the line emitting gas, consistent with conclusion (1) above. Correlations with EW(HeII) and the X-ray to optical spectral index further support this interpretation.

Progress on this line of approach to AGN variability will require longer light curves to obtain more accurate estimates of the variability properties, which are important to constrain both Poissonian and non-Poissonian models. Regardless of possible improvements on the time-series analysis techniques, it is important to encourage the continuation of the current CCD monitoring campaigns. Spectral information, some of which is already available (Kaspi et al. 2000), will also be valuable in deriving better constraints for the model parameters and a more detailed picture of the spectral behavior of the flares and background component.

The most appealing aspect of Poissonian models is their generality. Scenarios as diverse as accretion disk instabilities, stellar collisions and supernovae all fall under the large Poissonian "umbrella". Detailed modeling will be required to bridge the gap between this mathematical formalism and the physics of AGN variability and to fully 
explore the constraints made possible by the application of this technique.

It is a pleasure to thank the Wise Observatory group for their generous attitude of releasing to the scientific community the results of a laborious and lengthy observational project. We also thank T. Heckman and J. Krolik for their comments on an earlier version of this paper.
RCF thanks the hospitality of Johns Hopkins University, where this work was developed. Support for this work was provided by the National Science Foundation through grant \# GF-1001-99 from the Association of Universities for Research in Astronomy, Inc., under NSF cooperative agreement AST-9613615. LV acknowledges an MSc fellowship awarded by CAPES. Partial support from CNPq, FAPESP and PRONEX are also acknowledged.

\section{REFERENCES}

Abraham, Z., Romero, G. E. 1999, A\&A, 344, 61

Almaini, O., Lawrence, A., Shanks, T., Edge, A., Boyle, B. J., Georgantopoulos, I., Gunn, K. F., Stewart, G. C., Griffiths, R. E. 2000, MNRAS, 315, 325

Aretxaga, I., Cid Fernandes, R. \& Terlevich, R. 1997, MNRAS, 286, 271

Aretxaga, I., Terlevich, R. 1994, MNRAS, 269, 462

Ayal, S., Livio, M., Piran, T. 2000, ApJ, in press

Begelman, M., de Kool, M. 1991, in Variability of Active Galactic Nuclei, eds. H. R. Miller \& P. J. Wiita (Cambridge University Press), p. 198

Binette, L., Fosbury, R. A., Parker, D. 1993, PASP, 105, 1150

Bonoli, F., Braccesi, A., Federici, L., Zitelli, V., Formiggini, L. 1979, A\&AS, 35, 391

Boorgest, U., Schramm, K. J. 1994, A\&A, 284, 764

Cardeli, J. A., Clayton, G. C., Mathis, J. S. 1989, ApJ, 345, 245

Cid Fernandes, R. 1995, PhD Thesis, University of Cambridge

Cid Fernandes, R. 1997, Rev. Mex. Astron. Astrof., Conf. Series, 4, 210

Cid Fernandes, R., Aretxaga, I. \& Terlevich, R. 1996, MNRAS, 282, 1191

Courvoisier, T.J.-L., Paltani, S. \& Walter, R., 1996, A\&A, 308, L17

Cristiani, S., Vio, R., Andreani, P. 1990, AJ, 100, 56

Cristiani, S., Trentini, S., La Franca, F., Aretxaga, I., Andreani, P.

Vio, R., Gemmo, A. 1996, A\&A, 306, 395

Cristiani, S., Trentini, S., La Franca, F., Andreani, P. 1997, A\&A, 321,123

Cutri, R. M., Wiśniewski, W. Z., Rieke, G. H., Lebofsky, M. J. 1985, ApJ, 296, 423

Di Clemente, A., Giallongo, Natalo, G., Trevese, D., Vagnetti, F. 1996, ApJ, 463, 466

Edelson, R. A., Krolik, J. H., Pike, G. F.1990, ApJ, 359, 86

Edelson, R. A. et al. 1996, ApJ, 470, 364

Galeev, A. A., Rosner, R., Vaiana, G. S. 1979, ApJ, 229, 318

Garcia, A., Sodré, L., Jablonski, F. J. \& Terlevich, R. 1999, MNRAS, 309, 803

Giallongo, E., Trevese, D., Vagnetti, F. 1991, ApJ, 377, 345

Giveon, U., Maoz, D., Kaspi, S., Netzer, H., Smith, P. 1999, MNRAS, 306,637

Haardt, F., Maraschi, L., Ghisellini, G. 1994, ApJ, 432, L95

Hawkins, M. R. S. 2000, A\&AS, 143, 465

Hook, I. M., McMahon, R. G., Boyle, B. J., Irwin, M. J. 1994,

MNRAS, 268, 305

Lyutyi, V. M. 1977, SvA, 21, 655

Kaspi, S., Smith, P. S., Netzer, H., Maoz, D., Jannuzi, B. T. \& Giveon, U. 2000, ApJ, in press
Kawaguchi, T., Mineshige, S., Umemura, M., Turner, E. L. 1998, ApJ, 504, 671

Keenan, D. W. 1978, MNRAS, 185, 389

Kinney, A. L., Bohlin, R. C., Blades, J. C., York, D. G. 1991, ApJS, 75,645

Krolik, J. H., Horne, K. Kallman, T. R., Malkan, M. A., Edelson, R. A., Kriss, G. R. 1991, ApJ, 371, 541

Krolik, J. H., Done, C., Madejski, G. 1993, ApJ, 402, 432

Lehto, H. J. 1989, in Proceedings of the ESLAB Symposium, eds.

Hunt, J. \& Bottrick, B., (Paris:ESA), p. 499

Longo, G., Vio, R., Provenzale, A., Rifatto, A. 1996, A\&A, 312, 424

Maoz, D., Smith, P. S., Januzzi, B. T., Kaspi, S., Netzer, H. 1994,

ApJ, 421, 34

Mineshige \& Shields, G. 1990, ApJ, 351, 47

Netzer, H., Sheffer, Y. 1983, MNRAS, 203, 935

Netzer, H. et al. 1996, MNRAS, 279, 429

Netzer, H., Peterson, B. M. 1997, in Astronomical Time Series, eds. D. Maoz, A. Sternberg \& E. M. Leibowitz, (Kluwer Academic Publishers), p. 85.

Paltani, S., Walter, 1996, A\&A, 312, 55

Paltani, S., Courvoisier, T.J.-L. 1997, A\&A, 323, 717

Paltani, S., Courvoisier, T.J.-L., Walter, R. 1998, A\&A, 340, 47

Papoulis, A. 1965, Probability, random variables, and stochastic process, (McGraw-Hill)

Peterson, B. M. 1993, PASP, 105, 247

Peterson, B. M., Ferland G. 1986, Nature, 324, 345

Pica, A. J., Smith, A. G. 1983, ApJ, 272, 11

Pica, A. J., Smith, A. G., Webb, J. R., Leacock, R. J., Clemens, S., Gombola, P. P. 1988, AJ, 96, 1215

Press, W. H., Rybicki, G. B. 1997, in Astronomical Time Series,

eds. D. Maoz, A. Sternberg \& E. M. Leibowitz, (Kluwer Academic

Publishers), p. 61.

Schmidt, M., Green, R. E. 1983, ApJ 269, 352

Simonetti, J. H., Cordes, J. M., Heeschen, D. S. 1985, ApJ, 296, 46

Sirola, C. J., Turnshek, D. A., Weymann, R. J., Monier, E. M.,

Morris, S. L., Roth, M. R., Krzeminski, W., Kunkel, W. E., Duhalde,

O., Sheaffer, S. 1998, ApJ, 495, 659

Stern, B., Svensson, R., Sikora, M. 1991, in Variability of Active

Galactic Nuclei, eds. H. R. Miller \& P. J. Wiita (Cambridge

University Press), p. 229

Uomoto, A. K., Wills, B. J., Wills, D. 1976, AJ, 81, 905

Vieira, L. S. 2000, MSc Thesis, Universidade Federal de Santa

Catarina

Vio, R., Cristiani, S., Lessi, O., Provenzale, A. 1992, ApJ, 391, 518

Warner, B. 1995, Cataclysmic Variable Stars, Cambridge Astrophysics Series, Cambridge University Press, (Cambridge: New York)

\section{APPENDIX}

\section{SKEWNESS AND KURTOSIS IN POISSONIAN MODELS}

The Poissonian formalism allows the computation of higher moments of the $L(t)$ distribution, though most applications to AGN variability stop on the variance (eq. [7]). In this appendix we present expressions for the theoretically expected third (skewness) and fourth (kurtosis) moments, and discuss their applicability to the G99 data set. These moments were not used to constrain the flare properties of quasars in the main text, and are presented here only for the purposes of completeness and future reference.

The skewness $(\gamma)$ and kurtosis $(\kappa)$ are defined by

$$
\begin{gathered}
\gamma=\sigma^{-3 \overline{[L-\bar{L}]^{3}}} \\
\kappa=\sigma^{-4 \overline{[L-\bar{L}]^{4}}}-3
\end{gathered}
$$

The -3 term in (A2) makes the kurtosis of a Gaussian distribution 0. Expressions for $\gamma$ and $\kappa$ can be derived extending the formalism employed by Cid Fernandes (1995), which yields (Vieira 2000) 


$$
\begin{gathered}
\gamma=\phi_{\gamma}(\nu \tau)^{-1 / 2} \\
\kappa=\phi_{\kappa}(\nu \tau)^{-1}
\end{gathered}
$$

In these expression $\phi_{\gamma}$ and $\phi_{\kappa}$ play the role of "shape factors":

$$
\begin{aligned}
\phi_{\gamma} & =\frac{\tau^{2}}{E^{3}} \int l^{3}(t) d t \\
\phi_{\kappa} & =\frac{\tau^{3}}{E^{4}} \int l^{4}(t) d t
\end{aligned}
$$

Both $\phi_{\gamma}$ and $\phi_{\kappa}$ equal 1 for square shape flares. Exponential flares result in $\phi_{\gamma}=4 / 3$ and $\phi_{\kappa}=2$, while for triangular flares $\phi_{\gamma}=9 / 8$ and $\phi_{\kappa}=27 / 20$. The above relations reveal interconnections between the different moments in a Poissonian model: $\gamma \propto \delta$ and $\kappa \propto \delta^{2}$, where $\delta=\sigma / \bar{L}$ (eq. [7]). Unlike for $\delta$, an underlying non-variable component does not affect $\gamma$ nor $\kappa$. In principle, this allows an estimate of the background fraction from the ratio of $\gamma($ or $\kappa$ ) to $\delta$.

The main problem concerning the use of higher light curve moments is that, as can be seen in eqs. (A3) and (A4), the predicted moments rapidly tend to the Gaussian limit $(\gamma=\kappa=0)$ with increasing $\mathcal{N}=\nu \tau$, i.e., as the superposition of events grows higher. This is obviously a consequence of the Central Limit Theorem (e.g., Papoulis 1965).

The detection of deviations from Gaussianity in quasar light curves is known to be problematic (Press \& Rybicki 1997), and the G99 light curves are no exception. In Fig. A8 we present the skewness and kurtosis for the Wise Observatory data. Corrections for the effects of photometric errors upon the moments were applied, but were negligible, given the excellent accuracy of the G99 photometry. At first sight, the results in Fig. A8 would seem to immediately rule out any Poissonian model, as one finds negative $\gamma$ and $\kappa$ for about half of the objects, whilst the theory (eqs. [A3] and [A4]) predict only positive values!

This, however, is likely to be an effect of sampling. To demonstrate this, we have run a series of Poissonian light curve simulations and compared the output 2nd, 3rd and 4th moments with the predicted values as a function of observational parameters such as the length of the light curve $\left(T_{o b s}\right)$ and the number of observations $\left(n_{o b s}\right)$. The results clearly show that negative $\gamma$ and $\kappa$ occur very often also in simulated light curves, particularly for $T_{o b s} \lesssim 10 \tau$, as illustrated in Fig. A9. The scatter decreases for increasing $n_{o b s}$, but the agreement between analytical and simulated moments is only achieved for large $n_{o b s}$ and $T_{o b s} \gg \tau$. This bias is essentially insensitive to flare profile or their rate. The conclusion here is that one cannot use the currently available data to strongly constrain the higher moments of the $L(t)$ process. On the positive side, these experiments reinforce our conclusion that the actual flare shape is irrelevant for most of the analysis presented in this paper (see also next section).

We finalize by noting that, in analogy with what was done for the second moment, higher moments should be computed from their asymptotic $(\Delta t \rightarrow \infty)$ behavior estimated via SFs of the corresponding order. These, however, are subjected to large uncertainties due to the high powers involved and are not presented here.

\section{STRUCTURE FUNCTIONS FOR SIMPLE FLARE PROFILES}

The structure function of a Poissonian sequence of flares is:

$$
S F(\Delta t)=S F(\infty) s(\Delta t)
$$

where $S F(\infty)=2 \nu E^{2} / \tau$ is twice the asymptotic variance of the $L(t)$ process, and $s(\Delta t)$, the normalized SF of individual flares, is given by

$$
s(\Delta t)=1-\frac{\int l(t+\Delta t) l(t) d t}{\int l^{2}(t) d t}
$$

and is sensitive only to the shape of flare time profile $l(t)$.

\section{Square flares}

For $l(t)=l_{0}$ between $0<t<T$ and 0 otherwise one obtains a a linear SF up to $\Delta t=T$, with

$$
s(\Delta t)=\frac{\Delta t}{T}
$$

and 1 for $\Delta t>T$. The life-time $\tau$ (given by eq. 5 ) of square flares equals $T$. The dotted lines in Fig. B10 show the resulting $\mathrm{SF}$. 

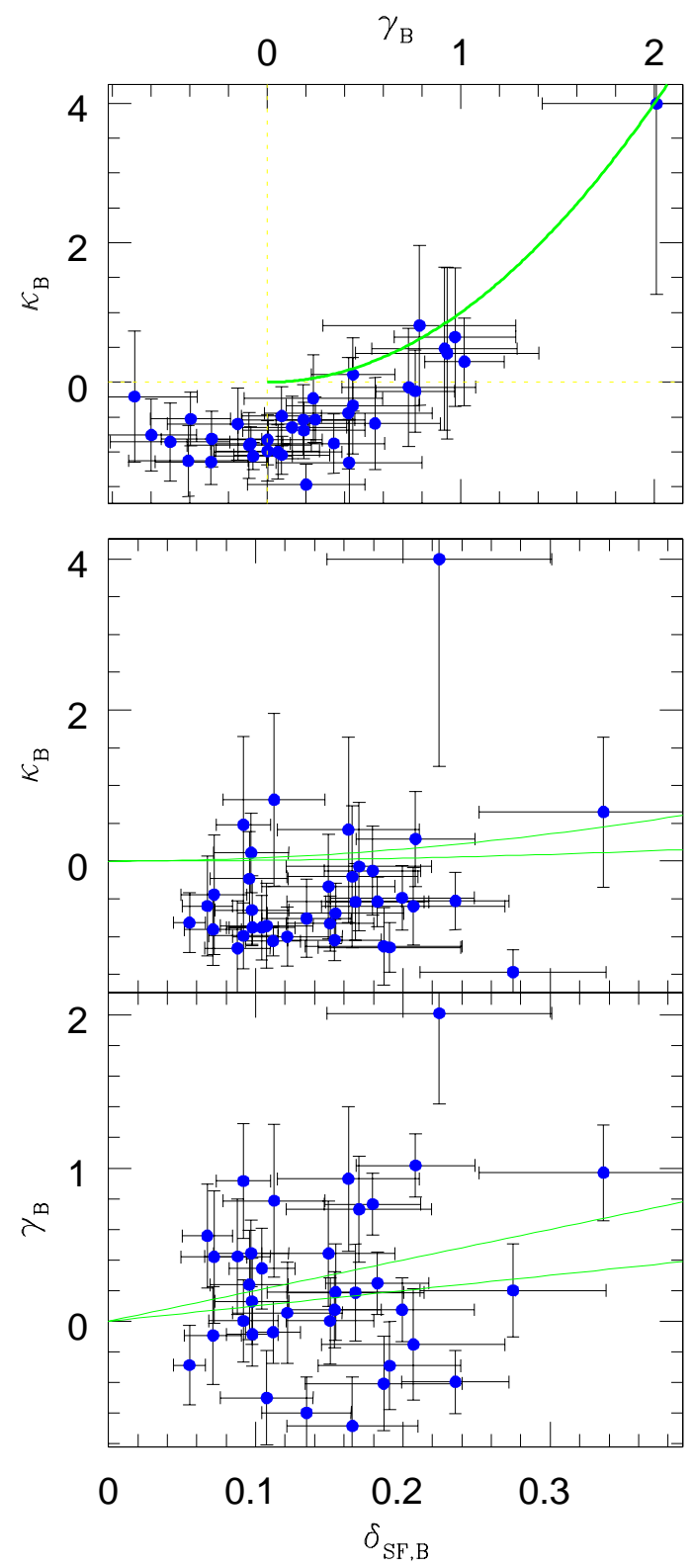

Fig. A8. - Skewness and kurtosis for the G99 B-band light curves plotted against each other (top panel), and $\delta_{S F}$ (bottom panels). Error bars for $\gamma$ and $\kappa$ were computed via bootstrap, resampling $n_{\text {obs }}$ randomly chosen points (with repetition) from the light curve 1000 times and computing the standard deviation of the resulting moments. The solid lines show the theoretical predictions for $\phi_{\gamma}=\phi_{\kappa}=1$ (square flares). In the bottom panels the two lines correspond predictions for a $c_{B}=50 \%$ background fraction (upper curves) and no background ( $c_{B}=0$, bottom curves). Note that $c$ affects only the abscissa in these plots, while the $\kappa-\gamma$ relation is independent of $c$.

\section{Exponentially decaying flares}

For $l(t)=l_{0} e^{-t / T}$ flares one finds

$$
s(\Delta t)=1-e^{-\Delta t / T}
$$

while the life-time is $\tau=2 T$. This $\mathrm{SF}$ is shown as a dot-dashed line in Fig. B10.

\section{Symmetric Triangular flares}

Linear flares with equal rise and decay times

$$
l(t)=l_{0} \times \begin{cases}(1+t / T) ; & \text { for }-T \leq t \leq 0 \\ (1-t / T) ; & \text { for } \quad 0 \leq t \leq T\end{cases}
$$

produce the following SF: 


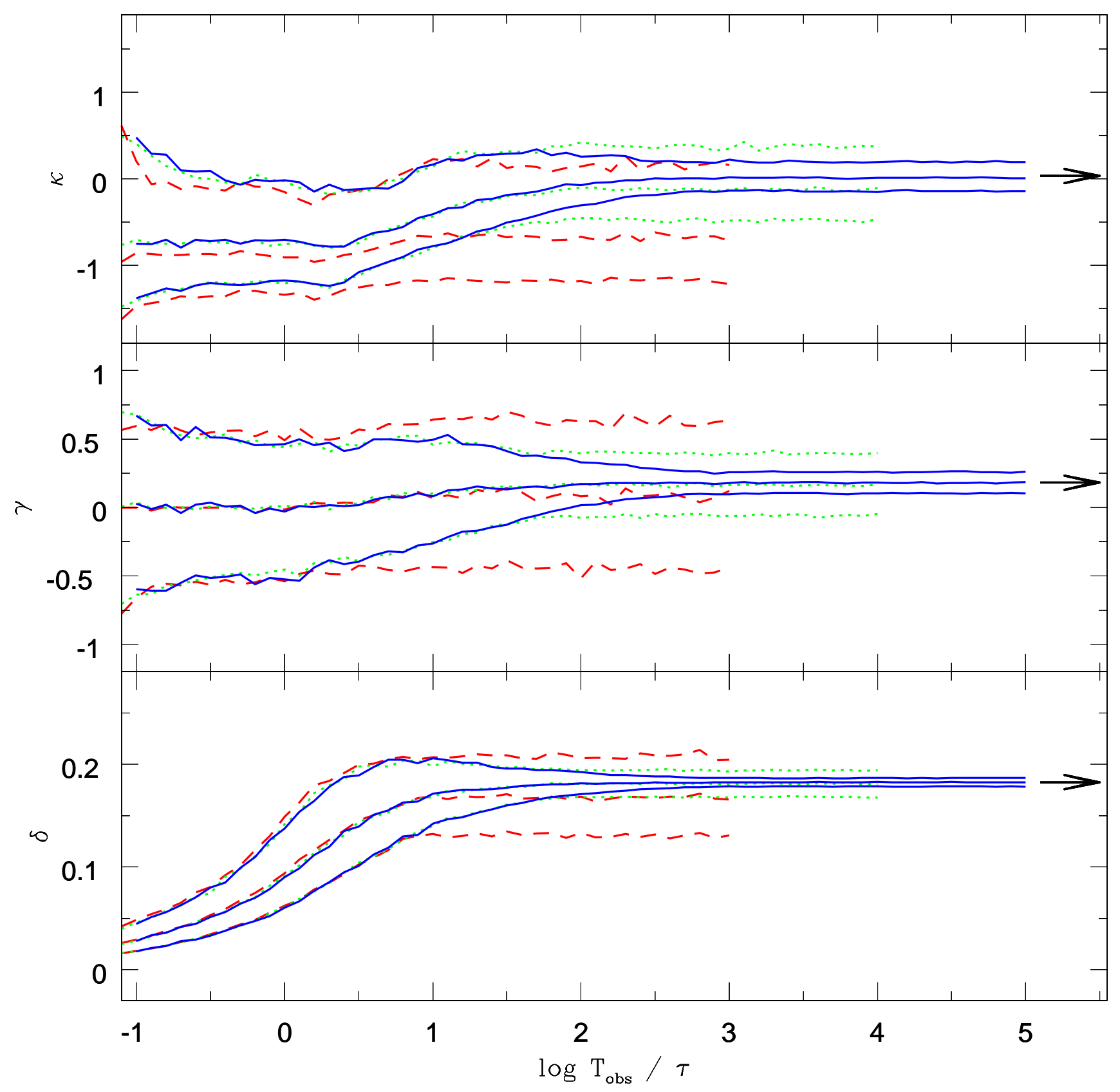

FIG. A9.- Fractional variability $(\delta)$, skewness $(\gamma)$ and kurtosis $(\kappa)$ for simulations as a function of the length of the light curve in units of $\tau$. Dashed, dotted and solid lines correspond to $n_{o b s}=11,101$ and 1001 observations respectively. The middle curves indicate the median over 1000 light curve simulations, whereas the bottom and top lines mark the 16 and $84 \%$ percentiles respectively, such that $68 \%$ of the points lie between them. Square flares and a rate of 30 events per $\tau$ were used in the simulations. The theoretical moments are indicated by the arrows on the right side of each panel. Large (and systematic in the cases of $\delta$ and $\kappa$ ) deviations from the expected values of the moments are observed for small $n_{o b s}$ and specially for short light curves $\left(T_{o b s} \lesssim 10 \tau\right)$.

$$
s(\Delta t)=\left\{\begin{array}{lr}
-\frac{3}{4}\left(\frac{\Delta t}{T}\right)^{3}+\frac{3}{2}\left(\frac{\Delta t}{T}\right)^{2} & ; \text { for } 0 \leq \Delta t \leq T \\
+\frac{1}{4}\left(\frac{\Delta t}{T}\right)^{3}-\frac{3}{2}\left(\frac{\Delta t}{T}\right)^{2}+3 \frac{\Delta t}{T}-1 & ; \text { for } T \leq \Delta t \leq 2 T \\
1 & ; \text { for } \quad 2 T \leq \Delta t
\end{array}\right.
$$

The relation between $\tau$ and $T$ for triangular shots is $\tau=3 T / 2$. The solid line in Fig. B10 shows this SF. 


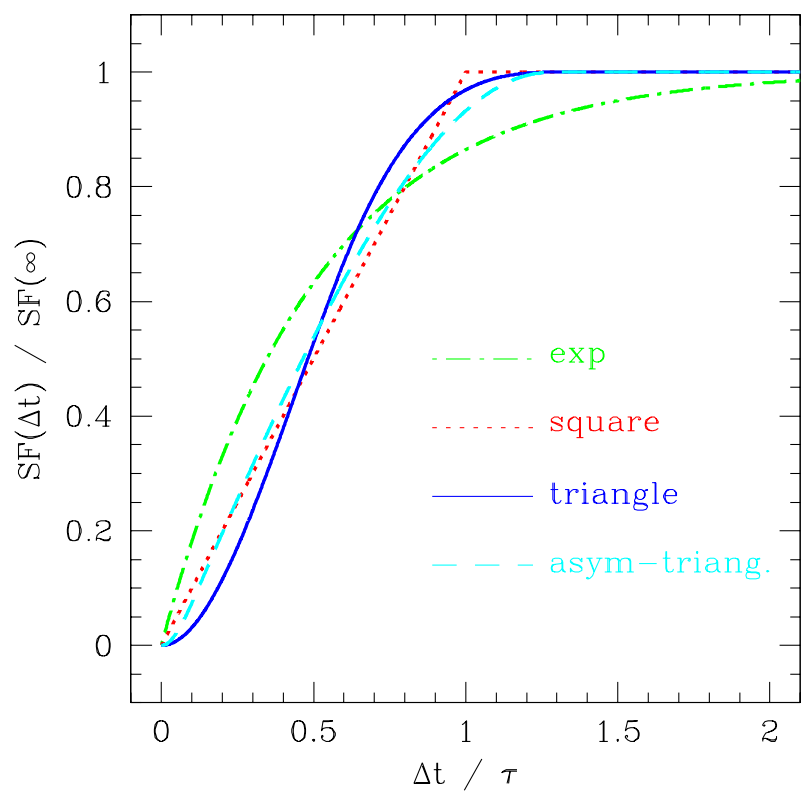

FIG. B10.- Theoretical Structure Functions for flares with square exponential, triangular and asymmetric triangle profiles.

Triangular flares with unequal rise $\left(T_{r}\right)$ and decay $\left(T_{d}\right)$ times

$$
l(t)=l_{0} \times\left\{\begin{array}{l}
\left(1+t / T_{r}\right) ; \text { for }-T_{r} \leq t \leq 0 \\
\left(1-t / T_{d}\right) ; \text { for } 0 \leq t \leq T_{d}
\end{array}\right.
$$

produce more complicated SFs: 


$$
s(\Delta t)= \begin{cases}-\frac{1}{2} \frac{\Delta^{3}}{T}\left(\frac{1}{T_{r}^{2}}+\frac{1}{T_{r} T_{d}}+\frac{1}{T_{d}^{2}}\right)+\frac{3}{2} \frac{\Delta^{2}}{T_{r} T_{d}} & ; \text { for } 0 \leq \Delta t \leq T_{r} \\ 1-\frac{1}{2} \frac{\Delta^{3}}{T T_{d}^{2}}+\frac{3}{2} \frac{\Delta}{T_{d}}-\frac{3}{2 T}\left(\frac{T_{r}^{2}}{6 T_{d}}+\frac{T_{r}}{2}+\frac{T_{d}}{3}\right) & ; \text { for } T_{r} \leq \Delta t \leq T_{d} \\ -\frac{1}{2}+\frac{1}{2} \frac{\Delta^{3}}{T T_{r} T_{d}}+\frac{3}{2} \frac{\Delta^{2}}{T_{r} T_{d}} & \\ +3 \frac{\Delta}{T}\left(1+\frac{T_{d}}{2 T_{r}}+\frac{T_{r}}{2 T_{d}}\right)-\frac{1}{2 T}\left(\frac{T_{d}^{2}}{T_{r}}+\frac{T_{r}^{2}}{T_{d}}\right) & ; \text { for } T_{d} \leq \Delta t \leq T \\ 0 & ; \text { for } T \leq \Delta t\end{cases}
$$

where $T=T_{r}+T_{d}$. The solution above corresponds to $T_{r}<T_{d}$. The corresponding expression for flares that spend more time rising than decaying is identical, with $T_{r}$ swapped by $T_{d}$. The life-time in either case is given by $\tau=3 T / 4$. G99 finding that quasars spend more time on the rise than fading gives some motivation to using $T_{r}>T_{d}$, but the SF, being a mean of squared differences, does distinguish between $T_{r}$ and $T_{d}$ (see Kawaguchi et al. 1998 for techniques to explore the time-assymetry of flares). Fig. B10 shows the SF for a ratio of 10 between $T_{r}$ and $T_{d}$ (or vice-versa) as a dashed line. 